\title{
Weighted voting, abstention, and multiple levels of approval ${ }^{1}$
}

\author{
J. Freixas* and W. Zwicker** \\ * Department of Applied Mathematics III \\ Polytechnic University of Catalonia \\ Av. Bases de Manresa, 61-73 \\ 08240 Manresa, Spain \\ ** Department of Mathematics \\ Union College \\ Schenectady, N.Y. 12308, USA
}

May 15, 2002

\begin{abstract}
In this paper we introduce the class of simple games with several ordered levels of approval in the input and in the output - the $(j, k)$ simple games - and propose a definition for weighted games in this context. Abstention is treated as a level of input approval intermediate to votes of yes and no. Our main theorem provides a combinatorial characterization, in terms of what we call grade trade robustness, of weighted $(j, k)$ games within the class of all $(j, k)$ simple games. We also introduce other subclasses of $(j, k)$ simple games and classify several examples. For example, we show the existence of a weighted representation for the UNSC, seen as a voting system in which abstention is permitted.
\end{abstract}

Key Words: Weighted voting, weighted majority, simple games, games with several levels of approval, trade robustness, abstention.

AMS subject classification: 91A12, 91A40, 91A80, 91C15

\section{Background and summary}

In modeling the type of voting intended to register collective approval or disapproval of a proposal, the literature on voting systems has confined itself almost exclusively to the mathematical structure known as a simple game (or simple voting game). In a simple game, a single alternative, such as a bill or an amendment, is pitted against the status quo, the players or voters vote in favor of the alternative or against it and the motion is passed or not depending of the collective strength of members who vote "yes." Abstention plays a key role in many of the real voting systems that have been modelled by these games (such as the United Nations Security Council, or the

\footnotetext{
${ }^{1}$ Research partially supported by Grant 1999BEAI400096 of the Commissioner for Universities and Research of the Catalonia Generalitat and by Grant BFM 2000-0968 of the Spanish Ministry of Science and Technology. The authors would like to thank Larry Becker, Clifford Brown, Vin Moscardelli, and Fred Jonas for their assistance. Comments of two anonymous referees greatly improved the manuscript.
} 
United States federal system), yet simple games, by their very nature, cannot take the possibility of abstention into account; those who do not vote "yes" are presumed to vote "no."

Indeed, Felsenthal and Machover have remarked on the extent to which some authors "mis-report the rules as though abstention were not a distinct option" (see [FM3], p. 22) and offer the hypothesis that "the misreporting is due to what philosophers of science have called theory-laden or theory-biased observation - a common occurrence, akin to optical illusion, whereby an observer's perception is unconsciously distorted so as to fit a preconception" (see [FM3], p. 280, as well as [FM1] and [FM2]).

We suspect that one factor that may have hindered the study of games with multiple levels of approval is the absence of a completely satisfactory definition of weighed voting in this context. Indeed, this gap partly explains our interest in the subject.

In the $(j, k)$ simple games we introduce here, each individual voter expresses one of $j$ possible levels of input support, and the output consists of one of $k$ possible levels of collective support. Standard simple games, then, are $(2,2)$ simple games, while $(3,2)$ simple games allow each voter a middle option, which may be interpreted as "I abstain." An important but isolated earlier work on abstention can be found in Fishburn (see [Fis1], pp. 53-55). Fishburn's context can be viewed as a special case of our $(j, k)$ games (in which $j=3=k$, the game is constant-sum, and the intermediate output level of approval is only achieved when the vote is tied exactly), and his definition of weighted game then coincides with the restriction of our definition to this special case. More recently, several works by Felsenthal and Machover (see [FM1], [FM2] and [FM3]) have been devoted to the study of voting systems with abstention. These authors argue the importance of taking abstention seriously, critique some justifications that others have offered for disregarding abstentions, and outline the rudiments of a theory of a priori voting power with abstention. Their "ternary voting rules" correspond to our $(3,2)$ simple games. In the alternative model proposed by Braham and Steffen ([BS]) abstention does not really figure as expressing an intermediate or even indeterminate degree of support between "yes" and "no." Instead, a sequential structure is proposed in which voters first choose between participation and abstention, and then those participating choose "yes" or "no." Lindner [Lin1] compares the Felsenthal and Machover approach to that of Braham and Steffan, and has also proposed in [Lin2] a model she calls multi-partition games, which resemble our $(j, 2)$ games. However, her model is fundamentally spatial, in that the voter inputs consist of points on the real line, and this leads her to a definition of weighted voting that is quite different from what we propose here.

In this paper, we treat an abstention as a level of input approval intermediate to a vote of yes and no. ${ }^{2}$ The United Nations Security Council and the US Congress (in each of

\footnotetext{
${ }^{2}$ Abstention may indicate indifference on the part of a voter, and indifference surely represents an intermediate level of approval. But a voter may abstain for a host of other reasons (such as absence from the voting chamber due to illness, a reluctance to be identified with either position, recusal due to a conflict of interest, etc.), many of which do not signal, in terms of the voter's intent, an intermediate level
} 
the two Houses) are two real-life examples that can be treated as what we call $(3,2)$ simple games; there are three levels of approval in the input, and two levels in the output. Once a third level of input approval is introduced, two further generalizations immediately suggest themselves: increasing the number of levels of approval in the input, and increasing the number of levels of approval in the output (outcome of the election). These are not empty generalizations. Our examples, in section 3 , of grading systems as $(j, k)$ games suggest that these multi-level structures arise naturally as models of certain types of decision making. Furthermore, Felsenthal and Machover have pointed out (see [FM3], p. 283) that the existence of composite voting games (in which the result of one vote is to inform a representative how to cast a vote in a larger election, in which other representatives are similarly informed by earlier votes) immediately suggests that abstain be a possible outcome or output of an earlier vote, if a representative is to be allowed to abstain in the larger vote.

Thus, our framework models voting systems for which the following conditions are met: (a) several levels of approval are permitted in the input, (b) several levels of approval are permitted in the output, and (c) those levels are qualitatively ordered. We call the resulting type of structure a $(j, k)$ simple game, where $j(k)$ denotes the number of input (output) levels in the game. As with ordinary simple games, the subclass of weighted $(j, k)$ games merits special consideration.

Classical cooperative games have given rise to several generalizations, related to our model, but distinguished in part by the fact that the output of a cooperative game is a cardinal value rather than a discrete level in a finite ordering. Bolger ([Bo1], [Bo2] and [Bo3]) deals with the so-called games with $n$ players and $r$ alternatives, in which the $r$ possible input alternatives are not ordered; each input alternative $j$ attracts its own coalition of supporting voters, and each such coalition is assigned an output cardinal value (so that the total output is an $r$-tuple of cardinal values). More recently, Magaña ([Mag]) and Amer et al. ([ACM]) have continued Bolger's work, introducing the closely related $r$-games, and including a model of the United Nations Security Council with abstention (quite different from ours, because of the $r$-tuple output values of Bolger's games). Hsiao and Raghavan ([HR]) consider multi-choice games, which are somewhat closer to our context: the inputs are ordered (each agent has an "effort level"), and the output is a single cardinal value. The primary concern of all these authors is in developing value theory (Shapley-Shubik or/and Banzhaf value) for the games they define. Each of these models admits a restriction that provides a different notion of "simple game," and some of our results about the structure of simple games with multiple levels of approval can be transferred to these other contexts. Our model is distinguished from all these others by the linear ranking of several discrete approval levels in both input and output, and our main concern in this paper is

of approval. However, in terms of the voter's effect on the outcome of the vote, an abstention is often treated (by the decision rule at hand), as if it represented an intermediate level of approval. An exception to this rule arises when a voting system includes a provision for a quorum. As we show later, in such a case it may prove impossible to identify an absence with an abstention, and impossible to totally order the inputs of yes, absent, and no in a satisfactory way. 
with matters other than value theory. It should be noted that all the "simple game" examples considered by those authors do entail a linear ranking in the input.

How should we define "weighted voting" for voting systems in which voters casts ballots that choose from among three or more possible levels of approval? In our view, this is the central question at hand.

Two possibilities suggest themselves immediately. In ratio-weighted voting, a proposal passes provided that the ratio between the total weight cast in favor to the total weight cast in favor or against (i.e., to the total weight of those who do not abstain) meet or exceed some threshold ratio. The rule for passage in difference-weighted voting is that the difference between the total weight cast in favor and that cast against meet or exceed some threshold difference. These possibilities differ from each other, although each reduces to the standard definition of weighted voting when we assume no voters abstain. Both assume (as the standard definition does) that each voter is assigned a single weight.

The definition we wish to propose is strictly more general than either of these, even when restricted to $(3,2)$ games. In particular, the reader may easily check that when abstentions are allowed, the UN Security Council is neither ratio- nor difference-weighted; in Section 5.1 we show that this system is weighted according to our broader definition. In choosing among possible definitions, we have been guided by three principles:

(a) the definition should be as general as possible,

(b) it should coincide with the ordinary notion of weighted majority game for $(2,2)$ games, and

(c) there should be a characterization of weighted voting in this context by a form of trade robustness that reduces to the standard characterization ([TZ1], [TZ2]) for $(2,2)$ games.

Any careful consideration of weighted voting in this broader context forces us to reexamine the standard definition of weighted voting for simple games. Recall that in a simple game $G=(N, W)$ each voter votes "yes" or "no," and the issue passes if and only if the coalition $X$ of yes-voting members of $N$ is a member of the set $W$ of "winning coalitions." In the standard definition of weighted voting for simple games, each "yes" voter $p$ contributes a certain nonnegative weight $w(p)$ to the total weight $S$ cast in favor, each "no" voter contributes 0 , and the issue is approved exactly if $S$ meets or exceeds some preset quota $q$.

But the following alternative definition seems just as reasonable: each "yes" voter contributes the weight $w_{y e s}(p)$ to the total weight $T$, each "no" voter contributes $w_{n o}(p)$ to $T$, and the issue passes exactly if $T$ meets or exceeds some preset quota $r$. That is, before any voting takes place each voter is pre-assigned two weights. We will also require that $w_{y e s}(p) \geq w_{n o}(p)$ for each voter $\mathrm{p}$, but will make no assumptions about the signs of $w_{y e s}(p)$ and $w_{n o}(p)$. 
Clearly, this alternative definition of "doubly-weighted" voting subsumes the original version as a special case, but is it actually any more general? Not at all - it turns out that only the differences $w_{y e s}(p)-w_{n o}(p)$ actually matter. In particular, if $w_{j}(Z)$ denotes the sum, over all $p$ in $Z$, of $w_{j}(p)$, and $X$ is the coalition of those voting "yes," then an issue passes under this second type of weighted voting

$$
\begin{array}{ll}
\text { iff } & w_{y e s}(X)+w_{n o}(N-X) \geq r \\
\text { iff } & w_{y e s}(X)-w_{n o}(N)+w_{n o}(N-X) \geq r-w_{n o}(N) \\
\text { iff } & w_{y e s}(X)-w_{n o}(X)-w_{n o}(N-X)+w_{n o}(N-X) \geq r-w_{n o}(N) \\
\text { iff } & w_{y e s}(X)-w_{n o}(X) \geq r-w_{n o}(N) .
\end{array}
$$

So if we set $w(p)=w_{y e s}(p)-w_{n o}(p)$ for each voter $p$, and define $q$ to be $r-w_{n o}(N)$, then our original doubly-weighted system is seen to be equivalent to standard weighted voting with the renormalized weights $w$ and quota $q$.

This very simple observation suggests a way to define weighted voting for the context wherein voters may choose from among several levels of approval. One obvious application is to voting systems with three input levels of approval: "yes", "abstain", and "no". In weighted voting with abstention, each "yes" voter contributes the weight $w_{y e s}(p)$ to the total weight $T$, each "abstain" voter contributes $w_{\text {abstain }}(p)$ to $T$, and each "no" voter contributes $w_{n o}(p)$ to $T$, with the issue passing exactly if $T$ meets or exceeds some preset quota $r$. That is, before any voting takes place each voter is pre-assigned three weights. We will also require that $w_{y e s}(p) \geq w_{\text {abstain }}(p) \geq w_{n o}(p)$ for each voter $p$, but will make no assumptions about the signs of $w_{\text {yes }}(p), w_{\text {abstain }}(p)$, or $w_{n o}(p)$.

Just as before, three weights represents superfluous information. If we renormalize by subtracting $w_{\text {abstain }}(p)$ from each of the weights $w_{y e s}(p), w_{\text {abstain }}(p)$, and $w_{\text {no }}(p)$ then the new triple of weights $w^{+}(p)=w_{\text {yes }}(p)-w_{\text {abstain }}(p), 0$, and $w^{-}(p)=w_{\text {no }}(p)-$ $w_{\text {abstain }}(p)$ describes the same voting system, and satisfies $w^{+}(p) \geq 0$ and $w^{-}(p) \leq 0$. There is no reason, in general, to suppose that $w^{-}(p)=-w^{+}(p)$, and every reason to allow these two quantities to be different, as in the case wherein some voters have veto power that is exercised through a "no" vote, but not through an abstention. Of course, it would be as easy to renormalize all "no" weights to zero, or all "yes" weights to zero, but the idea of abstention suggests that we renormalize at the middle level.

This explains why it seems appropriate to allow weighted voting with abstention to assign positive weights to "yes" votes, negative weights to "no" votes, to require no other relationship between a player's "yes" weight and their "no" weight (except in special cases, discussed in what follows) and to count abstentions as zero.

The organization of the paper is as follows. Section 2 has two parts, presenting the formal notions of $(j, k)$ simple games and of weighted $(j, k)$ games. Some subclasses of weighted $(j, k)$ games are also presented. In Section 3 some basic, introductory examples of $(j, k)$ simple games appear, among them the UNSC (United Nations Security Council) modeled as a $(3,2)$ simple game. In Section 4 , after presenting the notion of grade trade robustness, we provide the main theorem of the paper characterizing the 
weighted $(j, k)$ games in terms of the grade trade robustness property. The proofs for the $(j, 2)$ and $(2, k)$ cases, which require different arguments, are given here. In Section 5 , we discuss properties, including weightedness, for the games introduced in Section 3 ; in particular, we provide a very simple weighted representation for the UNSC as a $(3,2)$ simple game. Finally, an appendix includes the proof of the theorem for the general $(j, k)$ case with $j \geq 2$ and $k \geq 2$; roughly, this is a combination of the two particular cases treated in Section 4.

\section{$2(j, k)$ simple games}

Before the main notions are introduced we need some preliminary definitions. An ordered j-partition of the finite set $N$ is a sequence $A=\left({ }_{1} A, \ldots, j A\right)$ of mutually disjoint sets whose union is $N$. Any ${ }_{i} A$ is allowed to be empty, and we think of ${ }_{i} A$ as the set of those voters of $N$ who vote approval level $i$ for the issue at hand (where approval level 1 is the highest level of approval, 2 is the next highest, etc.). Notice that there is a natural one-to-one correspondence between 2-partitions (or bipartitions) and "yes"-voting coalitions; in this sense, an ordered $j$-partion of a $(j, k)$ simple game is analogous to a coalition of a standard simple game. Let ${ }^{j} N$ denote the set of all ordered $j$-partitions of $N$. For $X, Y \in{ }^{j} N$, we write $X^{j} \subseteq Y$ to mean that either $X=Y$ or $X$ may be transformed into $Y$ by shifting 1 or more voters to higher levels of approval. This is the same as saying ${ }_{i} X \uparrow \subseteq{ }_{i} Y \uparrow$ for each $i=1,2, \ldots, j$, where ${ }_{i} X \uparrow$ denotes ${ }_{1} X \cup$ ${ }_{2} X \cup \ldots \cup{ }_{i} X$ for each $i=1,2, . ., j$. We will use $X^{j} \subset Y$ to mean: both $X^{j} \subseteq Y$ and $X \neq Y$.

\subsection{Hypergraphs, prehypergraphs, games, and pregames}

Definition 2.1 $A(j, k)$ hypergraph $G=(N, V)$ consists of a finite set $N$ (the grand coalition of $G$ ) together with a value function $V:{ }^{j} N \rightarrow\left\{v_{1}, v_{2}, \ldots, v_{k}\right\}$; here $\left\{v_{1}, v_{2}, \ldots, v_{k}\right\}$ is the value set of $G$, whose members are any $k$ objects equipped with a strict linear ordering $v_{1}>v_{2}>$ $\ldots>v_{k}$. For ordered $j$-partitions $X$ and $Y$ we write $X<_{V} Y$ to mean $V(X)<V(Y)$, and $X \leq_{V} Y$ to mean $V(X) \leq V(Y)$.

Definition 2.2 $A(j, k)$ prehypergraph $G=(N, V)$ is a partially defined $(j, k)$ hypergraph; the domain of the value function $V$ is allowed to be a proper subset of ${ }^{j} N$.

The domain of a $(j, k)$ prehypergraph $G=(N, V)$ then refers to the domain of $V$, the members of $G^{\prime}$ 's domain are the classified $j$-partitions, and the remaining $j$-partitions are unclassified. In the context of $(j, k)$ prehypergraphs, $X<_{V} Y$ will mean that $X$ and $Y$ are classified with $V(X)<V(Y)$, and $X \leq_{V} Y$ will mean that $X$ and $Y$ are classified with $V(X) \leq V(Y)$. 
Definition 2.3 $A(j, k)$ simple game is a $(j, k)$ hypergraph that is monotonic: for all ordered $j$-partitions $X$ and $Y$, if $X^{j} \subseteq Y$ then $X \leq_{V} Y$.

An ordinary simple game may be identified with a $(2,2)$ simple game for which the value set is $\{$ win,lose $\}$ with win > lose, and a simple game with ties corresponds to a $(2,3)$ simple game with value set $\{$ win, tie, lose $\}$ and win $>$ tie $>$ lose.

Definition $2.4 A(j, k)$ pregame is a $(j, k)$ prehypergraph that is monotonic: for all classified ordered $j$-partitions $X$ and $Y$, if $X{ }^{j} \subseteq Y$ then $X \leq_{V} Y$.

The familiar notions of minimal winning coalition and maximal losing coalition for simple games may be extended to the context of $(j, 2)$ simple games: $X$ is a minimal winning $j$-partition provided that $X$ is winning and that for each $j$-partition $Y$ with $Y^{j} \subset X, Y$ is losing; $X$ is a maximal losing $j$-partition provided that $X$ is losing and that for each $j$-partition $Y$ with $X^{j} \subset Y, Y$ is winning. These notions in turn extend to $(j, k)$ games: a $j$-partition $X$ such that $V(X)=v_{i}$ is $i$-minimal for $i=1, \ldots, k-1$, if $V(Y)<v_{i}$ whenever $Y^{j} \subset X$. A $j$-partition $X$ such that $V(X)=v_{i}$ is $i$-maximal for $i=2, \ldots, k$, if $v(Y)>v_{i}$ whenever $X^{j} \subset Y$. These notions are useful in describing the game in a reduced form, and in studying weightedness via the algebraic approach of systems of linear inequalities; such inequalities compare the total weights of $i$-minimal $j$-partitions with those of $(i+1)$-maximal $j$-partitions for each $i=1, \ldots, k-1$. In some examples of Section 5 we will use this method to determine players' weights.

\subsection{Weighted $(j, k)$ games}

In the introduction, we argued in favor of assigning three weights to each player in any weighted voting systems with abstentions. This idea generalizes for $j$ levels of approval in the input, so that each player is assigned $j$ different weights. Renormalization would allow any one of these weights to be fixed for all voters at some specified value (such as zero), but different applications might suggest distinct renormalizations. The next definition also applies to games with multiple output levels.

Definition 2.5 Let $G=(N, V)$ be a $(j, k)$ prehypergraph (hypergraph). A representation of $G$ as a weighted $(j, k)$ prehypergraph (hypergraph) consists of a sequence $w=\left(w_{1}, \ldots, w_{j}\right)$ of $j$ weight functions, where $w_{i}: N \rightarrow \mathbb{R}$ for each $i$, together with $k-1$ real number quotas $q_{1} \geq q_{2} \geq \ldots \geq q_{k-1}$ such that for every classified $j$-partition $X$ and every $r$ with $1 \leq r \leq k$, $V(X)=v_{r}$ if and only if $q_{r-1}>w(X) \geq q_{r}$, where $w(X)$ denotes

$$
\sum\left\{\sum\left\{w_{i}(x) \mid x \in{ }_{i} X\right\} \mid 1 \leq i \leq j\right\}
$$

and we think of $q_{0}$ and $q_{k}$ as having nominal values of $+\infty$ and $-\infty$ respectively.

We say that $G=(N, V)$ is a weighted $(j, k)$ prehypergraph (hypergraph) if it has such a representation. 
Notice that if $G$ is weighted with weight function $w$, it follows that

$$
X>_{V} Y \Longrightarrow w(X)>w(Y)
$$

Furthermore, if there exists a weight function for which (1) holds (for each pair $X$, $Y$ of classified $j$-partitions), then $G$ is weighted in the sense of the definition above. For example, from (1) it follows for classified $j$-partitions $X$ and $Y$ that $w(X)>w(Y)$ holds whenever $V(X)=v_{1}$ and $V(Y)=v_{2}$, and from this we see that a quota $q_{1}$ may be inserted between the "lightest" classified $X$ with $V(X)=v_{1}$ and the "heaviest" classified $Y$ with $V(Y)=v_{2}$, etc.

Definition 2.6 Let $G=(N, V)$ be a $(j, k)$ pregame (simple game). A representation of $G$ as a weighted $(j, k)$ pregame (game) consists of a representation of $G$ as a weighted $(j, k)$ prehypergraph for which the weight functions satisfy the additional weight-monotonicity requirement that for each $x \in N, w_{1}(x) \geq w_{2}(x) \geq \ldots \geq w_{j}(x)$.

We say that $G=(N, V)$ is a weighted $(j, k)$ pregame (simple game) if it has a representation satisfying the weight-monotonicity requirement.

The next result will be used in the proof of the main theorem and states that if there exists a representation of a $(j, k)$ simple game $G$ as a weighted $(j, k)$ hypergraph, and this representation fails to satisfy the additional weight-monotonicity requirement of the previous definition, then there exists a different representation for the game that meets this condition. Formally,

Theorem 2.7 Let $G$ be a $(j, k)$ simple game that is weighted as a $(j, k)$ hypergraph. Then $G$ is a weighted $(j, k)$ simple game; that is, if $u_{1}, u_{2}, \ldots, u_{j}$, together with $k-1$ associated real quotas $q_{1} \geq q_{2} \geq \cdots \geq q_{k-1}$ is a representation of $G$ as a weighted $(j, k)$ hypergraph, then the $j$ weight functions can be adjusted to satisfy

$$
u_{1}(x) \geq u_{2}(x) \geq \ldots \geq u_{j}(x) \text { for each } x \in N .
$$

Proof: Let $u_{1}, u_{2}, \ldots, u_{j}$ be a weighted representation of $G$ that does not satisfy the weight-monotonicity requirement, so that for some voter $x \in N, u_{l}(x)<u_{l+1}(x)$ with $1 \leq l \leq j-1$. Consider a new weighted representation $v$ for $G$ defined by

$$
v_{i}(p)= \begin{cases}u_{l+1}(p) & \text { if } p=x, i=l \\ u_{l}(p) & \text { if } p=x, i=l+1 \\ u_{i}(p) & \text { otherwise }\end{cases}
$$

Then $v_{l}(x)>v_{l+1}(x)$. For every $j$-partition $A$ with $x \in{ }_{l+1} A$ consider the $j$-partition $B$ obtained from $A$ by shifting the voter $x$ to the immediately higher level of approval $l$. Then by our assumption,

$$
A \leq_{V} B \quad \text { and } \quad u(A)>u(B)
$$


Let $1 \leq r \leq k$ be such that $q_{r-1}>u(A) \geq q_{r}$. Then from (3) it follows $q_{r-1}>u(A)>$ $u(B) \geq q_{r}$, and as

$$
\begin{aligned}
& v(A)=u(A)+u_{l}(x)-u_{l+1}(x)=u(B) \\
& v(B)=u(B)+u_{l+1}(x)-u_{l}(x)=u(A)
\end{aligned}
$$

it then follows that

$$
q_{r-1}>u(A)=v(B)>v(A)=u(B) \geq q_{r} .
$$

This process may be repeated as needed until condition (2) is satisfied for each $x \in N$. Notice that the proof relies on the assumption that all $j$-partitions are classified ( $G$ is a game rather than a pregame). To establish the result for pregames (if, indeed, this generalization is valid), would require quite a different argument.

There is a special subclass of weighted games that deserves mention.

Definition 2.8 $A(j, k)$ simple game is strongly weighted if there is a total order $\succeq$ on $N$ and a weighted representation such that if $r \succeq p$, then $w_{i}(r)-w_{i+1}(r) \geq w_{i}(p)-w_{i+1}(p)$ for each $p, r \in N$ and every $i=1, \ldots, j-1$.

In $(3, k)$ games (for instance, in games with abstention) there is a subclass of strongly weighted games that can be represented by weights such that:

$$
w_{1}(p)=-w_{3}(p) \text { and } w_{2}(p)=0
$$

Definition 2.9 If a $(3, k)$ game admits a weighted representation as in (4) we shall say that $G$ is a zero-centered strongly weighted game.

If $k=3$ we consider a still more restrictive subclass, for which $q_{2}=-q_{1}$ and $q_{1}>0$ is taken to be small enough to satisfy that for every 3-partition $A$, if $w(A)>0$ then $w(A)>q_{1}$.

Definition 2.10 When $G$ admits this special type of representation we will say that $G$ is a relative majority game.

In a relative majority game, each player $p$ has, in effect, a single nonnegative weight $u(p)$ that is cast positively when they vote in favor of an issue and is cast negatively when they vote against, with no weight cast when they abstain. The issue passes if strictly more total weight is cast in favor than is cast against and fails if this inequality is reversed, with a tie obtaining when the total weight cast in favor exactly balances that cast against:

$$
V(A)=V\left({ }_{1} A,{ }_{2} A,{ }_{3} A\right)= \begin{cases}\text { win } & \text { if } u\left({ }_{1} A\right)>u\left({ }_{3} A\right) \\ \text { lose } & \text { if } u\left({ }_{1} A\right)<u\left({ }_{3} A\right) \\ \text { tie } & \text { otherwise }\end{cases}
$$


where $u\left({ }_{i} A\right)$ means $\sum_{p \in{ }_{i} A} u(p)$ for each $i=1,2,3$. Thus, relative majority games have a constant-sum property as well as a knife-edge property: if all voters reverse their votes (with the reverse of "yes" being "no" and the reverse of "abstain" being "abstain") then the outcome is reversed (with the reverse of "pass" being "fail" and the reverse of "tie" being "tie"), and any switch by any single non-dummy voter (one whose weight is non-zero) breaks a tied outcome.

These relative majority games correspond exactly to the weighted games with abstention that were considered by Fishburn (see [Fis1]), and constitute a slight variation on the relative majority $r$-games considered by Magaña (see [Mag]). Notice that a particular subclass of these games, obtained when $u\left({ }_{i} A\right)=\left|{ }_{i} A\right|$ for $i=1,3$, models the case of relative majority when the game is symmetric: all voters all have the same influence (with one vote for each voter).

If we focus on the class of $(3,2)$ games, how does our definition of "weighted voting with abstentions" compare with the earlier-mentioned ratio and difference notions? According to Definition 2.6, if we renormalize to make $w_{\text {abstain }}(p)=0$ for each voter $p$ then the rule for passage in a weighted $(3,2)$ game is

$$
V\left({ }_{1} A,{ }_{2} A,{ }_{3} A\right)=\text { win iff } w_{\text {yes }}\left({ }_{1} A\right)+w_{\text {no }}\left({ }_{3} A\right) \geq q .
$$

For ratio-weighted voting, this becomes

$$
V\left({ }_{1} A,{ }_{2} A,{ }_{3} A\right)=\text { win iff } u\left({ }_{1} A\right) \geq r\left[u\left({ }_{1} A\right)+u\left({ }_{3} A\right)\right],
$$

where $u$ is the single weight function and $r$ is the threshold ratio. This is equivalent to

$$
V\left({ }_{1} A,{ }_{2} A,{ }_{3} A\right)=\text { win iff } u\left({ }_{1} A\right)-r^{\prime} u\left({ }_{3} A\right) \geq 0,
$$

where $r^{\prime}=r /(1-r)$. For difference-weighted voting, the rule is

$$
V\left({ }_{1} A,{ }_{2} A,{ }_{3} A\right)=\text { win iff } u\left({ }_{1} A\right)-u\left({ }_{3} A\right) \geq d,
$$

where $u$ is the single weight function and $d$ is the threshold difference. Clearly, (7) is a special case of (5) (in which $w_{n o}(p)=-r^{\prime} w_{y e s}(p)$ for each voter $p$, and $q=0$ ), and (8) is also a special case of (5) (in which $w_{n o}(p)=-w_{y e s}(p)$ for each voter $p$, and $q=d$ ).

These expressions become simpler if we restrict our attention to symmetric games, in which each player has the same influence (the automorphism group of the game is the full symmetric group), so that we can assume, without loss of generality, that there exists a nonnegative constant $s$ such that for every voter $p$, $w_{y e s}(p)=u(p)=1$, and $w_{n o}(p)=-s$. The Definition 2.6 version (5) then becomes

$$
V\left({ }_{1} A,{ }_{2} A,{ }_{3} A\right)=\text { win iff }\left|{ }_{1} A\right|-s\left|{ }_{3} A\right| \geq q,
$$

the ratio-weighted version reduces to

$$
V\left({ }_{1} A,{ }_{2} A,{ }_{3} A\right)=\text { win iff }\left|{ }_{1} A\right|-\left.r^{\prime}\right|_{3} A \mid \geq 0,
$$


and the difference-weighted requirement simplifies to

$$
V\left({ }_{1} A,{ }_{2} A,{ }_{3} A\right)=\text { win iff }\left|{ }_{1} A\right|-\left|{ }_{3} A\right| \geq d .
$$

Notice that for symmetric games, Definition 2.6 thus reduces to the obvious common generalization of ratio- and difference-weightedness. ${ }^{3}$

An equivalent definition of weightedness may be phrased by assigning weights to the "steps" between levels rather than to the levels. For a $(j, k)$ game $G=(N, V)$ in which there are $j$ levels of approval in the input, there are $j-1$ steps from the highest level to the lowest.

Consider a $(j, k)$ prehypergraph $G$ for which each player $p$ is assigned one weight for each step, yielding $j-1$ step weights $d_{1}(p), d_{2}(p), \ldots, d_{j-1}(p)$; if $G$ is a pregame or game rather than a prehypergraph then we require each $d_{i}(p) \geq 0$.

Given a $j$-partition $A=\left({ }_{1} A, \ldots, j A\right)$ the total weight $w(A)$ is defined as the sum, over each $r=1,2, \ldots, j-1$ and each player $p$ in ${ }_{r} A$, of the quantity

$$
\sum_{h=r}^{j-1} d_{h}(p) .
$$

Now, we define $G$ to be weighted iff

$$
V(A)=v_{r} \Longleftrightarrow q_{r-1}>w(A) \geq q_{r}
$$

for each $1 \leq r \leq k$ and every classified $j$-partition $A$. We note that this latter definition is equivalent to the former one, with each step weight corresponding to the difference between weights for successive levels. For a strongly weighted game with $r \succeq p$ we have $d_{i}(r) \geq d_{i}(p)$ for every $i=1, \ldots, j-1$, and for a zero-centered strongly weighted $(3, k)$ game we may take $d_{1}(p)=d_{2}(p)$.

\section{Some examples}

In this section we describe some examples of $(j, k)$ simple games that later will be classified according to the different notions of simple games given above. The main tool to study their weightedness will be the characterization theorem of Section 4 .

\footnotetext{
${ }^{3}$ In [List] Christian List considers symmetric games (such as those modeling juries) in which there exists an objectively "correct" outcome, and for which our voting system is intended to guarantee a certain minimum probability that the outcome of the group vote is correct. He argues in favor of the difference definition over the ratio definition, for this context. His conclusions are intended to apply when the same decision rule applies as the number of voters varies, but in his context this variation is unrelated to abstentions.
} 


\subsection{The United Nations Security Council as a simple game and as a $(3,2)$ simple game}

The United Nations Security Council (UNSC) has 10 nonpermanent members and 5 permanent members. At least 9 affirmative votes are needed to pass a motion, subject to a veto by any of the 5 permanent members. In the standard model of the UNSC as a simple game, a coalition $X$ is winning if and only if all permanent members and at least four of the nonpermanent members belong to $X$ (vote in favor). It is well known that while not every simple game is weighted, this UNSC game admits the weighted representation:

$$
[39 ; 7,7,7,7,7,1,1,1,1,1,1,1,1,1,1]
$$

Shapley and Shubik in [SS] used essentially this model (but at a time when the Council had only 6 nonpermanent members, for a total of 11) to calculate the voting power (in terms of what is now called the Shapley-Shubik index) of the members, Straffin (in [Str], pp. 314-315) used the model to find voting power via the Banzhaf index, and a number of other authors have referred to this standard model when discussing the UNSC.

The rules for voting in the UNSC were set down in Article 27 of the UN Charter, which reads as follows (with the material in brackets indicating the amendations made in 1965, when the Council was enlarged):

1. Each member shall have one vote.

2. Decisions of the Security Council on procedural matters shall be made by an affirmative vote of seven [nine] members.

3. Decisions of the Security Council on all other matters shall be made by an affirmative vote of seven [nine] members including the concurring votes of the permanent members; provided that, in decisions under Chapter VI, and under paragraph 3 of Article 52, a party to a dispute shall abstain from voting.

Do these rules allow a permanent member to abstain, and if so does such an abstention necessarily block passage of a non-procedural motion? In [Fas], pages 179-181, Fassbender argues, on several grounds, that the original intent of the language and its initial interpretation is that absence or abstention of a permanent member is tantamount to a veto. Others have argued that "affirmative" and "concurring" have different meanings, and that a permanent member may indicate concurrence by abstaining. For a bibliography of work on this issue, see [Sim].

Regardless of the original wording, however, there is no doubt that in practice a permanent member can actively abstain or can choose not to participate in a vote or can be absent, and these actions are not interpreted as vetos of non-procedural motions. ${ }^{4}$

\footnotetext{
${ }^{4}$ Fassbender refers to this change in Article 27 as an "informal amendment," and points out that it was
} 
Perhaps the most famous precedent ocurred in 1950, when the USSR boycotted the Council in protest over who would control China's seat (see [Fas], p. 178). When the remaining Council members voted in favor of sending UN forces to Korea (an action which the USSR strongly opposed), the USSR's absence did not prevent passage of the motion. There are more recent examples, as well. In one case, resolution 344 passed on December, 1973, with all five permanent members abstaining.

The simple game model, presented earlier, is thus inadequate, because it neglects the possibility of abstention, in effect identifying, for any permanent member of the Council, not voting "yes" with the casting of a veto. Clearly, to decide whether a motion passes in the United Nations Security Council, one must know how the members voted from among each of the three alternatives "yes," "abstain" and "no" - the UNSC voting system should be properly modelled as a $(3,2)$ simple game. ${ }^{5}$ In fact, Bolger ([Bo2]) used the UNSC to illustrate his extension of the Shapley value to games with alternatives.

Because a resolution is carried in the Security Council if at least nine members support it and no permanent member is explicitly opposed, our formal description of the UNSC as a $(3,2)$ game is as follows: Let $P=\{1,2,3,4,5\}$ and $R=\{6,7, \ldots, 15\}$ be the sets of permanent members and nonpermanent members respectively, and

$$
V(A)=V\left({ }_{1} A,{ }_{2} A,{ }_{3} A\right)= \begin{cases}\text { win } & \text { if }\left|{ }_{1} A\right| \geq 9 \text { and }{ }_{3} A \cap P=\emptyset \\ \text { lose } & \text { otherwise }\end{cases}
$$

Here, we think of ${ }_{1} A$ as the set of those voters of $N$ who vote "yes, ${ }_{2} A$ as the set of those voters of $N$ who abstain, and ${ }_{3} A$ as the set of those voters of $N$ who vote "no."

\subsection{Three voting rules for the United States Senate}

Decisions in the two houses of the United States Congress are governed by a variety of voting rules, depending on the nature of the issue at hand. We will focus, somewhat arbitrarily, on a few different examples from the US Senate, which consists of 100 Senators together with the vice-president of the United States (who leads the Senate and casts tie-breaking votes in certain circumstances). ${ }^{6}$ In some cases, these rules appear in the Constitution of the United States, while in others they have evolved (and are subject to further change by the Senate); ${ }^{7}$ some of these evolved rules appear in the

"in line with the wishes of the overwhelming majority of the members of the international community," in part because "abstention made it possible for a permanent member to express its reservations about a particular decision while not obstructing it." He quotes the US delegate to the General Assembly, Senator Tom Connally, as arguing just this point on November 15, 1946 (see [Fas], p 181-182)

${ }^{5}$ We treat both absence and choosing not to participate in a vote as forms of abstention, as they have the same effect.

${ }^{6}$ The United States Constitution states that "The Vice President of the United States shall be President of the Senate, but shall have no Vote, unless they be equally divided."

${ }^{7}$ One way for a minority of Senators to block passage of a bill is to mount a "filibuster," in which they speak for indefinite length, for example by reading names from a telephone directory. After a rule 
Standing Rules of the Senate (at http://rules.senate.gov/senaterules/menu.htm).

The three voting systems we discuss here each have an accompanying quorum, or minimum number of senators who must be present in order for the senate to do business. However, rules for quorums are somewhat complex, so we begin by ignoring quorums in these system, taking up this matter again at the end of this section.

\subsubsection{Passing an ordinary bill or amendment}

Passing a bill requires a simple (strict) majority of those who vote "yes" or "no," except that when the number of "yes" and "no" votes are exactly equal, the vice president casts a tie-breaking vote. For example, if 81 senators abstain or are absent, 10 vote in favor of a bill, and 9 senators vote against, the bill passes (regardless of whether or not the vice president is also opposed and brings the total "no" vote up to 10). ${ }^{8}$

This system is modeled as a $(3,2)$ simple game in which

$$
V_{B}(A)=V_{B}\left({ }_{1} A,{ }_{2} A,{ }_{3} A\right)= \begin{cases}\text { win } & \text { if }\left|{ }_{1} A\right|>\left|{ }_{3} A-\{v p\}\right| \\ \text { lose } & \text { otherwise }\end{cases}
$$

Here " $v p$ " stands for the vice-president.

\subsubsection{Ratifying a treaty}

The US Senate must ratify any treaty with a foreign government before that treaty becomes law. Ratification requires that two thirds of those senators present must vote in favor of the treaty. For example, if 90 senators are present, at least 60 must vote in favor. In particular, if 58 of these 90 vote in favor, 28 vote against, and 4 abstain, then the treaty is not ratified. Note that in this system, an abstention by a senator who is present is equivalent to a vote of "no," but such an abstention is not equivalent to an absence (because if the four abstainers in the example had stayed away, the treaty would have been ratified). The vice president does not get to vote in this situation, because the "equally divided" language of the Constitution does not apply to supermajorities.

change in 1917, the senate was able to break a filibuster by invoking "cloture," which cuts off any further debate on a bill, provided that two thirds of the full membership of the senate (67 Senators) vote in favor of cloture. In 1975 the requirement was changed to require only three fifths of the Senate (60 Senators).

${ }^{8}$ Suppose that there is an exact tie among senators, perhaps with 50 favoring a bill and 50 opposed, and the vice-president then abstains. We have found no written rules stating what would happen (perhaps because the situation does not arise in practice, as the administration takes a position on most bills). But it seems most likely that the bill would fail to pass in this situation, as it would in the House (where there is no one to break ties, and the edge is given to the status quo body of law - that is, no change is made to the body of law in case of an exact tie vote over whether to change that body of law by approving a new bill), and so this feature is built into our game model. Evidence is provided by vice president Gore's tie-breaking vote on the budget in 1993. Gore ran in order to make it to the Senate in time to vote to enact the budget; he wouldn't have needed to do so if a 50-50 tie had meant enactment. 
As abstention and absence are inequivalent, it is not so clear how to model the voting system as a $(j, k)$ simple game. We've chosen to treat the system as a $(4,2)$ simple game in which

$$
V_{T}(A)=V_{T}\left({ }_{1} A,{ }_{2} A,{ }_{3} A,{ }_{4} A\right)= \begin{cases}\text { win } & \text { if }\left|{ }_{1} A-\{v p\}\right| \geq 2\left|\left({ }_{3} A \cup{ }_{4} A\right)-\{v p\}\right| \\ \text { lose } & \text { otherwise }\end{cases}
$$

Here, we intend ${ }_{3} A$ to contain those voters who are present but abstain, and ${ }_{2} A$ to contain the absent voters. An odd feature of this model is that a treaty is passed if no senators are present. A modification that defeats the treaty in this (highly unlikely) scenario is given by $V_{T^{\prime}}$ :

$V_{T^{\prime}}(A)=V_{T^{\prime}}\left({ }_{1} A,{ }_{2} A,{ }_{3} A,{ }_{4} A\right)= \begin{cases}\text { win } & \text { if } 0 \neq\left|{ }_{1} A-\{v p\}\right| \geq 2\left|\left({ }_{3} A \cup{ }_{4} A\right)-\{v p\}\right| \\ \text { lose } & \text { otherwise }\end{cases}$

(An alternative approach, perhaps just as good, would be to treat the system as a $(3,2)$ simple game by ignoring the possibility of voters who are present but abstain, because such voters might as well have voted "no.")

\subsubsection{Electing a vice president when the electoral college fails to do so}

The twelfth amendment of the constitution states that in the event that the electoral college has failed to elect a vice president (because no single candidate has received a majority of electoral votes) the Senate shall elect the vice president, choosing between the two candidates achieving the highest numbers of votes in the electoral college. To win this Senate election, a candidate must receive at least 51 votes. ${ }^{9}$ There is no explicit mention, in this amendment, of what happens if neither candidate receives 51 votes (because some senators abstain or are absent); we will treat this as an intermediate outcome, "inconclusive." 10 The (current) vice president can serve as the fifty-first vote in this type of election. ${ }^{11}$

\footnotetext{
${ }^{9}$ The relevant passage reads: “The person having the greatest number of votes as Vice-President shall be the Vice-President, if such number be a majority of the whole number of electors appointed, and if no person have a majority, then from the two highest numbers on the list, the Senate shall choose the Vice-President; a quorum for the purpose shall consist of two-thirds of the whole number of Senators, and a majority of the whole number shall be necessary to a choice."

${ }^{10}$ In practice it seems very likely that senators would attend and not abstain in such a situation. Also, the Senate can continue to hold votes until one of the two candidates does receive 51 votes.

${ }^{11}$ Our model presumes that if either candidate receives 50 votes, the vice president can become the fifty-first vote, regardless of how many votes are received by the other candidate. However, the "evenly divided" language in the Constitution, together with the "majority of the whole number" phrase in Amendment XII, suggests that perhaps the tie-breaking power of the (current) vice-president only applies in a Senate election for the (next) vice-president when there are exactly 50 votes for each candidate (as opposed, for example, to a 50-47 vote with three senators absent or abstaining). If so, our model would need to be modified, but not in any way that would affect the proof, in section 5.2, that the game is not weighted. After the 2000 election for president and vice president, the previously elected vice president Albert Gore presided, in January of 2001, over a newly elected Senate with exactly 50 Republicans
} 
Suppose that the two candidates with the greatest number of electoral votes are $a$ and $b$. Then the voting system can be treated as a $(3,3)$-game in which $a$ 's supporters form the coalition ${ }_{1} A$, $b$ 's supporter's form ${ }_{3} A$, abstainers form ${ }_{2} A$, the value set contains the three levels $a$ wins $>$ inconclusive $>b$ wins, and

$$
V_{v p}(A)=V_{v p}\left({ }_{1} A,{ }_{2} A,{ }_{3} A\right)= \begin{cases}a & \text { if }\left|{ }_{1} A\right| \geq 51 \\ \text { inconclusive } & \text { if }\left|{ }_{1} A\right|<51 \text { and }\left|{ }_{3} A\right|<51 \\ b & \text { if }\left|{ }_{3} A\right| \geq 51\end{cases}
$$

At first, it seems that the ordered levels of approval inherent to $(j, k)$ games may be appropriate in modeling the Senate vice-presidential election. After all, supporters of candidate $a$ (a left-winger, let us suppose) would probably regard the input "abstain" as intermediate to supporting $a$ and supporting $b$ (the standard bearer of the right wing), and those in $b^{\prime}$ 's camp would agree. And while $b^{\prime}$ s supporters might reverse the ordering of inputs and outputs seen as natural by $a^{\prime}$ 's supporters, this sort of reversal is ubiquitous in situations modeled by $(j, k)$ games. However, there are some strong counterarguments, ${ }^{12}$ that cast doubt on whether ordered levels of approval are quite apt for this voting system (see also some remarks in the Conclusion).

Each of the above systems is subject to the requirement that a quorum (minimum number) of members be present in order for the Senate to conduct business. This quorum is ordinarily 51 senators, ${ }^{13}$ but for ratification of a treaty the quorum is set at 67 . However, the number of senators present is not officially counted unless some member actually calls for such a count, and legislation does pass at times when everyone is well aware that they are far short of a quorum. ${ }^{14}$

and 50 Democrats. The split in the electoral college (actually 271 for Bush and 267 for Gore) could easily have been exactly 269-269, had a few close states gone the other way. In this case, a very strange scenario would become quite plausible: Gore would have been able to break a 50-50 tie in the Senate and elect his own Democrat vice-presidential running mate, Lieberman (who, as a senator, would presumably have voted for himself), while at the same time the Republican controlled House would in all likelihood have elected Bush as president.

${ }^{12}$ For example, imagine that there are three parties fielding vice presidential candidates and all three are represented in the Senate. Consider the senators from the party supporting the candidate $c$ obtaining the third highest number of electoral college votes. If these senators choose to vote neither for $a$ nor for $b$, this choice need not represent a position intermediate to supporting $a$ and supporting $b$. Perhaps this explains why, as we see, in section 5.2, this particular voting system fails to be weighted, despite its evident simplicity. An alternate (3, 2)-simple game model could be based on the idea that the Senate first votes ("yes," "no," or "abstain") on some one of these candidates (say, a), who wins if the "yes" votes total 51 or more, and loses otherwise (which makes abstentions effectively equal to "no" votes). Then, if $a$ fails to be elected, they vote on $b$ using the same voting system. The final outcome is presumably the same as with the original system (assuming $b$ 's supporters vote "no" when $a$ is voted on, and vice-versa), and this alternative game is easily seen to be weighted, but this alternative does not seem very true to the actual way such a vote would (presumably) be held.

${ }^{13}$ The constitution defines a quorum as a majority of the members, but the Standing Rules of the Senate define a quorum as a majority of the senators, so apparently the vice president does not contribute to a quorum.

${ }^{14}$ In fact, the Speaker of the House of Representatives has to "hear" a call for such a count. If he is intentionally deaf that day, the demand for a quorum call is in vain. Of course, the Speaker cannot abuse this privilege too often. 
If a senator calls for a quorum count, and it is discovered that the count falls short, then a majority of the senators present can compel attendance by the others, instructing the sergeant at arms to go out and round them up. If he fails to do so within one hour, then the Senate must discontinue doing business (unless the original senator retracts her or his request for a quorum count, which is sometimes done when the original purpose was to produce a small delay).

However, if business is suspended on account of lack of a quorum, the result is that the bill or amendment under discussion at the time of suspension is taken up again when the Senate reconvenes. The effect, then, is to force postponement, but not to defeat the bill.

As the reader may already suspect, the complexities in these rules make it very difficult to take account of quorums in our formal model. Indeed, because the effect of calling for a quorum is, at most, that no vote actually takes place (rather, the vote is delayed) one can argue that the role of quorums should not properly be incorporated in the voting system at all. Indeed, this is the position we favor.

However, if one does attempt to build in the effect of quorums, then in the context of $(j, k)$ games there seem to be no reasonable alternatives to the following three assumptions:

1. Quorum counts are "automatic": a bill cannot pass the Senate unless 51 Senators or more are present.

2. If there are fewer than 51 Senators present, the result is "postponement," rather than defeat of the bill, and postponement is an output level intermediate between passage and defeat.

3. Abstention and absence represent different levels of input approval (because abstainers who are present contribute to the quorum count, while absent members do not).

Based on these assumptions, we suggest the following model. Notice that the added input level of "absent" and the added output level of "postponement" imply that a Senate voting system with quorum incorporated is likely to be modeled as a $(4,3)$ game.

\subsubsection{Passing an ordinary bill or amendment, with quorum incorporated}

Suppose that the "yes" voters belong to ${ }_{1} A$, the abstainers belong to ${ }_{2} A$, those absent belong to ${ }_{3} A$, and the "no" voters belong to ${ }_{4} A$. Then our model is defined by

$$
V_{B Q}\left({ }_{1} A,{ }_{2} A,{ }_{3} A,{ }_{4} A\right)= \begin{cases}\text { win } & \text { if }\left|{ }_{1} A\right|>\left|{ }_{4} A-\{v p\}\right| \text { and }\left|{ }_{3} A-\{v p\}\right| \leq 49 \\ \text { postpone } & \text { if }\left|{ }_{3} A-\{v p\}\right|>49 \\ \text { lose } & \text { otherwise }\end{cases}
$$


The most striking aspect of this model is its failure to be monotonic. If 50 senators favor a bill, 1 opposes it, and 49 are absent, then the bill will pass. But had the voter in opposition chosen, instead, to be absent, the bill would have been postponed; by increasing her input level of approval, this voter would lower the output level - a failure of monotonicity. Notice that in a different scenario, a similar switch from "no" to "absent" can cause a bill that was voted down to be postponed (or even to pass), so that such a switch has neither a consistently positive nor a consistently negative effect on the outcome of the vote. ${ }^{15}$

\subsection{A $(4,2)$ simple game}

This example is taken from Hsiao and Raghavan ([HR], page 241). In a mathematics department with 50 professors $(N=\{1, \ldots, 50\}), 10$ are distinguished $(D=\{1, \ldots, 10\})$. Each professor may express strong support, marginal support, weak opposition, or strong opposition to the proposed promotion of a certain junior colleague. The candidate will be promoted only under one of the following scenarios:

1. There are at least 40 professors showing their marginal or strong support, and furthermore at least two distinguished professors are not strongly opposed.

2. At least 25 professors strongly support the candidate, and furthermore at least one among them is a distinguished professor.

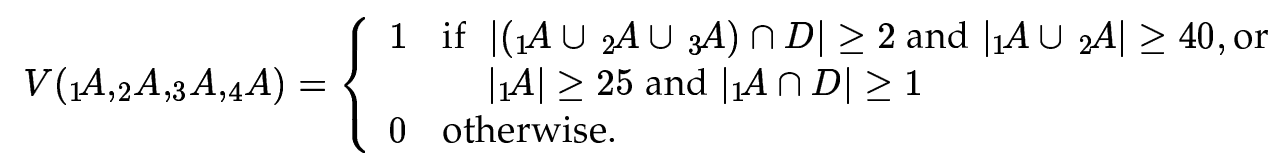

This is a game with 50 players and four levels of approval in the input. Hsiao and Raghavan ([HR]) define a suitable power index to measure each professor's influence over the final outcome, while our interest is in studying the representability of this game as a weighted voting system.

\subsection{Two grading systems}

Grading systems provide natural examples of $(j, k)$ games with larger values of $j$ and $k$. We give two specific examples here, reflecting common practices in the United States and in Spain, respectively.

The grades or marks assigned to undergraduate students at Union College are similar to those in use at many colleges and universities in the United States. Each student receives a final course grade of $\mathrm{A}, \mathrm{A}-, \mathrm{B}+, \mathrm{B}, \mathrm{B}-, \mathrm{C}+, \mathrm{C}, \mathrm{C}-, \mathrm{D}$, or $\mathrm{F}$ in each course

\footnotetext{
${ }^{15}$ Similarly, it is easy to see that switching between "abstain" and "absent" can have an inconsistent effect on the outcome. For this reason, our decision to interpret "abstain" as a higher approval level than "absent" was a somewhat arbitrary one.
} 
taken by the student, with A representing the top of the scale. These grades have standard numerical equivalents, assigned by the college registrar, as follows:

\begin{tabular}{|c|c|c|c|c|c|c|c|c|c|}
\hline $\mathrm{A}$ & $\mathrm{A}-$ & $\mathrm{B}+$ & $\mathrm{B}$ & $\mathrm{B}-$ & $\mathrm{C}+$ & $\mathrm{C}$ & $\mathrm{C}-$ & $\mathrm{D}$ & $\mathrm{F}$ \\
\hline 4.0 & 3.7 & 3.3 & 3.0 & 2.7 & 2.3 & 2.0 & 1.7 & 1.0 & 0.0 \\
\hline
\end{tabular}

These numbers are used to calculate each students cumulative index (the average of all their course grades), but may also be used to calculate the final grade in a single course, when several grades are assigned during the course of the term. For example, suppose that in a certain Mathematics class there are 4 quizzes, 2 tests, and a final examination, for a total of 7 grades, that are weighted differently in calculating the final course grade, according to the following grading scheme (announced by the instructor on the first day of classes):

1. each quiz contributes $5 \%$ of the final course grade,

2. each test contributes $20 \%$ of the final course grade, and

3. the final examination contributes $40 \%$ of the final course grade.

Suppose further that Sara's grades in this class are as follows:

$\mathrm{B}-, \mathrm{C}, \mathrm{A}-$, and $\mathrm{B}-$ (quiz grades); $\mathrm{B}-$ and $\mathrm{B}+$ (test grades); $\mathrm{A}-$ (final examination grade).

Sara's final numerical average for the course is the weighted sum of the numerical equivalents for these grades:

$$
\begin{aligned}
& (0.05)(2.7)+(0.05)(2.0)+(0.05)(3.7)+(0.05)(2.7) \quad \text { (quiz grades) } \\
& +(0.20)(2.7)+(0.20)(3.3) \quad \text { (test grades) } \\
& +(0.40)(3.7) \text { (final examination grade). } \\
& =3.237 \text {. }
\end{aligned}
$$

The translation of this numerical average $w$ into one of the 10 possible letter course grades is up to the discretion of the instructor, but one reasonable method is to place thresholds midway between the numerical equivalents, as follows:

\begin{tabular}{|c|c|c|c|c|c|}
\hline if $w$ satisfies & $w \geq 3.85$ & $3.5 \leq w<3.85$ & $3.15 \leq w<3.5$ & $\ldots$ & $w<0.5$ \\
\hline then final grade is & $\mathrm{A}$ & $\mathrm{A}-$ & $\mathrm{B}+$ & $\ldots$ & $\mathrm{F}$ \\
\hline
\end{tabular}

In particular, Sara's final course grade is B+.

Such a grading scheme corresponds to a weighted $(10,10)$ game, in which each single quiz, test, or exam corresponds to a single voter, and the issue being voted on by these 7 voters is, "Should Sara get a high grade in this course?" Each "voter" is assigned 10 weights, corresponding to the 10 possible grades that voter might assign to Sara. In the case of the example we have been discussing, these weights consist of the numerical equivalents of the 10 grades, each multiplied by the percentage assigned by the 
grading scheme to quizzes $(0.05)$, tests $(0.20)$ or exams $(0.40)$. For example

$$
\begin{array}{lll}
w_{1}(\text { quiz } 1)=(0.05)(4.0), & w_{2}(\text { quiz } 1)=(0.05)(3.7), & \text { etc., } \quad \text { while } \\
\left.w_{1} \text { (test } 1\right)=(0.20)(4.0), & \text { etc. }
\end{array}
$$

Finally, the quotas for this weighted $(10,10)$ game are the midpoint thresholds mentioned before:

$$
\left(q_{1}, q_{2}, \ldots, q_{7}, q_{8}, q_{9}\right)=(3.85,3.5, \ldots, 1.85,1.35,0.5)
$$

The last step in obtaining the engineering degree at the Polytechnic School of Manresa is an evaluation of each student's final project. The grading system for this evaluation is similar to those in use at other schools in the Polytechnic University of Catalonia.

The academic committee is formed by three professors who evaluate students. Each professor evaluates a different aspect: one assesses the theoretical contents, another the laboratory training, and the last considers the exercises written by the student. Each student then is assigned a single, final mark compounded from the separate marks proposed by the three professors. The possible final marks that a student can get are: excellent $\left(v_{1}\right)$, notable or creditable $\left(v_{2}\right)$, pass $\left(v_{3}\right)$, and fail $\left(v_{4}\right)$. The possible marks that a professor may assign are: right (right votes are clustered in ${ }_{1} A$ ), regular (regular votes are clustered in ${ }_{2} A$ ) and wrong (wrong votes are clustered in ${ }_{3} A$ ), and the rule that determines final marks is given by the following $(3,4)$ game:

$V\left({ }_{1} A,{ }_{2} A,{ }_{3} A\right)= \begin{cases}\text { excellent } & \text { if pra } \in{ }_{1} A,\left|{ }_{1} A\right| \geq 2 \text { and }{ }_{3} A=\emptyset \\ \text { notable } & \text { if }{ }_{3} A=\emptyset, \text { and }{ }_{1} A=\{\text { pra }\} \text { or }{ }_{2} A=\{\text { pra }\} \\ \text { pass } & \text { if }\left|{ }_{1} A\right|=1,\left|{ }_{2} A\right|=2, \text { pra } \in{ }_{2} A ; \text { or }\left|{ }_{1} A\right|=2,{ }_{2} A=\emptyset, \text { th } \in{ }_{1} A \\ \text { fail } & \text { otherwise }\end{cases}$

wherein "th" and "pra" refer, respectively, to the theory professor and to the practical professor.

\section{A characterization theorem for $(j, k)$ weighted games.}

What is it that makes a $(j, k)$ simple game weighted or not? One approach to this question for ordinary $(2,2)$ simple games made use of the following observation: If $G$ is a weighted game then $G$ is "trade robust," meaning that if a collection of winning coalitions from $G$ engage in an exchange or "trade" of players, then at least one of the coalitions is still winning after the trade. As is shown in Theorem 2.4.2 of Taylor and Zwicker [TZ2], page 57, as generalized in footnote 9 on the same page, trade robustness is also sufficient to guarantee the existence of weights: 
Theorem 4.1 Characterization Theorem for Weighted Prehypergraphs. For a prehypergraph $G=(N, W, L)$, the following are equivalent:

(i) $G$ is weighted.

(ii) $G$ is trade robust.

(iii) $G$ is $2^{2^{|N|}}$-trade robust.

Here $N$ denotes the set of voters, $W$ the set of winning coalitions, and $L$ the set of losing coalitions.

\subsection{The characterization for $(j, 2)$ games}

Our goal, then, is to generalize the trading characterization to $(j, k)$ simple games. We do this in three steps. In this section, we state and prove the characterization for the $(j, 2)$ case. In the following section, we give the characterization for the general $(j, k)$ case and provide the proof for the $(2, k)$ case. The proof of the general case, essentially a combination of these two arguments, is given in the appendix.

First, however, we seek the analogue, in $(j, k)$ games, to the notion of a trade in ordinary simple games. In a standard simple game, if any single player $p$ moves out of one of the coalitions in the sequence $\left\langle X_{1}, X_{2}, \ldots, X_{m}\right\rangle$ and into another, she trades her presence in the former coalition for her absence in the latter. Such a switch has no net effect on $\left|\left\{i \mid p \in X_{i}\right\}\right|$. Correspondingly, if $\left\langle X_{1}, X_{2}, \ldots, X_{m}\right\rangle$ is a sequence of $j$-partitions, a single player $p$ might trade her support at level $s$ in one of the $j$-partitions for her support at level $t$ in another. Such a switch has no net effect on $\left|\left\{i \mid p \in{ }_{r} X_{i}\right\}\right|$ for any $r$ with $1 \leq r \leq j$, and this suggests the following definition:

Definition 4.2 We will say that one sequence $\left\langle X_{1}^{\prime}, X_{2}^{\prime}, \ldots, X_{m}^{\prime}\right\rangle$ of $j$-partitions has been obtained from another sequence $\left\langle X_{1}, X_{2}, \ldots, X_{m}\right\rangle$ of the same length $m$ via trades, providing that $\left|\left\{i \mid p \in{ }_{r} X_{i}\right\}\right|=\left|\left\{i \mid p \in{ }_{r} X_{i}^{\prime}\right\}\right|$ for each $p \in N$ and each $r$ with $1 \leq r \leq j$.

It is straightforward (see Proposition 2.3 .2 on page 56 of [TZ2]), if tedious, to show that if one sequence is obtained from another via trades, then the transformation can in fact be effected through a sequence of trades or switches: in any single switch a player $p$ moves from level $s$ in the $i^{t h} j$-partition up to level $t$ in the $i^{\text {th }} j$-partition, while at the same time the same individual moves from level $t$ in the $e^{t h} j$-partition down to level $s$ in the $e^{t h} j$-partition.

We state the trade robustness notion for $(j, 2)$ simple games, and then the characterization theorem.

Definition $4.3 A(j, 2)$ prehypergraph $G$ is trade robust if whenever $m \geq 1$ is an integer, $\left\langle X_{1}, X_{2}, \ldots, X_{m}\right\rangle$ is a sequence of classified $j$-partitions, and a second sequence $\left\langle X_{1}^{\prime}, X_{2}^{\prime}, \ldots, X_{m}^{\prime}\right\rangle$ of classified $j$-partitions is obtained from the first via trades, it is not the case that all the $X_{1}, X_{2}, \ldots, X_{m}$ are winning and all the $X_{1}^{\prime}, X_{2}^{\prime}, \ldots, X_{m}^{\prime}$ are losing. 
Theorem 4.4 Let $G$ be any $(j, 2)$ prehypergraph (simple game). Then $G$ is a weighted $(j, 2)$ prehypergraph (simple game) if and only if $G$ is trade robust.

Proof: $(\Rightarrow)$ Assume $G=(N, V)$ is weighted, let $\left\langle X_{1}, X_{2}, \ldots, X_{m}\right\rangle$ be a sequence of winning $j$-partitions, and let $\left\langle X_{1}^{\prime}, X_{2}^{\prime}, \ldots, X_{m}^{\prime}\right\rangle$ be a second sequence of classified $j$-partitions obtained from the first via trades. Note that the sum $w\left(X_{1}\right)+\ldots+w\left(X_{m}\right)$ can be rewritten as the sum of all terms of form

$$
\left(w_{r}(p)\right)\left|\left\{i \mid p \in{ }_{r} X_{i}\right\}\right|
$$

taken over all $p \in N$ and all $r$ with $1 \leq r \leq j$. As

$$
\left|\left\{i \mid p \in{ }_{r} X_{i}\right\}\right|=\left|\left\{i \mid p \in{ }_{r} X_{i}^{\prime}\right\}\right|
$$

is true for all $p \in N$ and all $r$ with $1 \leq r \leq j$, it follows that

$$
w\left(X_{1}^{\prime}\right)+\ldots+w\left(X_{m}^{\prime}\right)=w\left(X_{1}\right)+\ldots+w\left(X_{m}\right) .
$$

If $w\left(X_{l}^{\prime}\right)=\max \left\{w\left(X_{i}^{\prime}\right) \mid 1 \leq i \leq m\right\}$, then we have

$$
w\left(X_{l}^{\prime}\right) \geq \frac{w\left(X_{1}^{\prime}\right)+\ldots+w\left(X_{m}^{\prime}\right)}{m}=\frac{w\left(X_{1}\right)+\ldots+w\left(X_{m}\right)}{m} \geq \frac{q \cdot m}{m}=q .
$$

Thus, $X_{l}^{\prime}$ is winning and $G$ is trade robust.

$(\Leftarrow)$ Assume $G=(N, V)$ is trade robust. We will define an auxiliary prehypergraph $G^{*}$, and prove that $G^{*}$ is trade robust. Then we will apply the fundamental Theorem 2.4.2 of [TZ2] to $G^{*}$ to obtain weights for $G^{*}$, and use these weights for $G$.

For the grand coalition of $G^{*}$, we will need $j$ disjoint copies of the original grand coalition $N=\{1,2, \ldots, n\}$ of $G$. For each $l$ with $1 \leq l \leq j$ let $N^{l}$ denote $\left\{1_{l}, \ldots, n_{l}\right\}$ (our "copy l" of $N$ ), and let $N^{*}=N^{1} \cup \cdots \cup N^{j}$ be the grand coalition for $G^{*}$. For any coalition $X \subseteq N^{*}$ and $l$ with $1 \leq l \leq j$, let $X_{(l)}$ denote $\left\{i \in N \mid i_{l} \in X\right\}$. We will say that $X \subseteq N^{*}$ is a partition code if the $X_{(l)}$ are pairwise disjoint and $X_{(1)} \cup X_{(2)} \cup \cdots \cup X_{(j)}=N$.

Consider now the prehypergraph defined on $N^{*}$ by the function $V^{*}$ for which:

$V^{*}(X)= \begin{cases}\text { win } & \text { if } X \text { is a partition code and } V\left(X_{(1)}, X_{(2)}, \ldots, X_{(j)}\right)=\text { win }, \\ \text { lose } & \text { if } X \text { is a partition code and } V\left(X_{(1)}, X_{(2)}, \ldots, X_{(j)}\right)=\text { lose } \\ \text { unclassified } & \text { otherwise }\end{cases}$

Now, we will prove that if $G$ is trade robust then $G^{*}$ is trade robust. Suppose, by way of contradiction, that $G^{*}$ were not trade robust. Then we could find winning coalitions $X_{1}, X_{2}, \ldots, X_{m} \subseteq N^{*}$ in the pregame $G^{*}$ together with trades that convert them into losing coalitions $Y_{1}, Y_{2}, \ldots, Y_{m} \subseteq N^{*}$. Consider the corresponding $j$-partitions, 


$$
\begin{gathered}
\left\{\begin{array}{l}
\left(\left(X_{1}\right)_{(1)},\left(X_{1}\right)_{(2)}, \ldots,\left(X_{1}\right)_{(j)}\right) \\
\left(\left(X_{2}\right)_{(1)},\left(X_{2}\right)_{(2)}, \ldots,\left(X_{2}\right)_{(j)}\right) \\
\ldots, \\
\left(\left(X_{m}\right)_{(1)},\left(X_{m}\right)_{(2)}, \ldots,\left(X_{m}\right)_{(j)}\right)
\end{array}\right. \\
\left\{\begin{array}{l}
\left(\left(Y_{1}\right)_{(1)},\left(Y_{1}\right)_{(2)}, \ldots,\left(Y_{1}\right)_{(j)}\right) \\
\left(\left(Y_{2}\right)_{(1)},\left(Y_{2}\right)_{(2)}, \ldots,\left(Y_{2}\right)_{(j)}\right) \\
\ldots, \\
\left(\left(Y_{m}\right)_{(1)},\left(Y_{m}\right)_{(2)}, \ldots,\left(Y_{m}\right)_{(j)}\right)
\end{array}\right.
\end{gathered}
$$

All of the $X j$-partitions are winning in $G$ and all of the $Y j$-partitions are losing in $G$, and the $Y j$-partitions are obtained via trades from the $X j$-partitions, contradicting $G^{\prime}$ s presumed trade robustness.

Thus, $G^{*}$ is a trade robust prehypergraph. It then follows (by Theorem 2.4 .2 of [TZ2]), that $G^{*}$ is weighted as a prehypergraph, and so we can realize it as:

$$
G^{*} \equiv\left[q ; w_{1}(1), \ldots, w_{j}(1), w_{1}(2), \ldots, w_{j}(2), \ldots, w_{1}(n), \ldots, w_{j}(n)\right]
$$

where $w_{l}(i)$ is the weight assigned to the $i_{l}$ member in $N^{*}$ for each $1 \leq l \leq j$ and $1 \leq i \leq n$. Then, a representation of $G$ as a weighted $(j, 2)$ prehypergraph is given by $q$ and the sequence $w=\left(w_{1}, \ldots, w_{j}\right)$ of $n$ weight functions. Finally, note that if $G$ is a $(j, 2)$ simple game ( $G$ is monotonic and all $j$-partitions are classified), then we can adjust, by Theorem 2.7, the weight functions to satisfy that for each $x \in N$, $w_{1}(x) \geq w_{2}(x) \geq \ldots \geq w_{j}(x)$.

\subsection{The characterization for $(j, k)$ games, and proof for the $(2, k)$ case}

Here, we define grade trade robustness for $(j, k)$ prehypergraphs, which serves as the analogue of trade robustness for $(j, 2)$ games but comes in two equivalent forms, and then phrase the corresponding theorem in terms of these notions. These definitions closely resemble the known combinatorial characterizations for additively representable (also called additively separable) orderings of the powerset of a finite set. ${ }^{16}$

\footnotetext{
${ }^{16} \mathrm{An}$ ordering of sets is additively representable if it is possible to assign numerical weights to the members of these sets in such a way that the ordering is that induced by total weight. This notion differs from what we consider here, because with additive representability there is no finite sequence of quotas that "lump" coalitions at the same output level, provided that the gap between their weights fails to contain one of the separating quotas. The question of characterizing additively representable orderings first arose in the work of de Finetti [deF] in subjective probability theory. The problem was solved by Kraft, Pratt, and Seidenberg [KPS], and has since been reformulated and simplified by others (see, for example, the survey article by Fishburn, [Fis2]). If the strict inequalities in our definition of grade trade robust are adjusted, so that the first $m-1$ are made into weak inequalities (while the final inequality remains strict), then the resulting definition coincides with the characterization of additively representable orderings on subsets of a finite set.
} 
Definition $4.5 A(j, k)$ prehypergraph $G$ is grade trade robust if whenever $m \geq 1$ is an integer, $\left\langle X_{1}, X_{2}, \ldots, X_{m}\right\rangle$ is a sequence of classified $j$-partitions, and a second sequence $\left\langle X_{1}^{\prime}, X_{2}^{\prime}, \ldots, X_{m}^{\prime}\right\rangle$ of classified $j$-partitions is obtained from the first via trades, it is not the case that $X_{i}>_{V} X_{i}^{\prime}$ for each $i=1,2, \ldots, m$.

We will say that $G$ is grade ${ }^{+}$trade robust if whenever $m \geq 1$ is an integer, $\left\langle A_{1}, A_{2}, \ldots, A_{m}\right\rangle$ and $\left\langle B_{1}, B_{2}, \ldots, B_{m}\right\rangle$ are equal length sequences of classified $j$-partitions satisfying that $\left.A_{i}\right\rangle_{V}$ $B_{i}$ for each $i=1,2, \ldots, m$ and $\left\langle A_{1}^{\prime}, A_{2}^{\prime}, \ldots, A_{m}^{\prime}\right\rangle$ is obtained from the original $A$ sequence via trades, and $\left\langle B_{1}^{\prime}, B_{2}^{\prime}, \ldots, B_{m}^{\prime}\right\rangle$ is obtained from the original $B$ sequence via trades, it is not the case that $A_{i}^{\prime}<_{V} B_{i}^{\prime}$ for each $i=1,2, \ldots, m$.

Proposition 4.6 $A(j, k)$ prehypergraph $G$ is grade trade robust if and only if it is grade ${ }^{+}$ trade robust.

Proof: Suppose that $G$ is not grade trade robust, and let

$$
X_{i}>_{V} X_{i}^{\prime}
$$

(for each $i=1,2, \ldots, m$ ) witness a failure of $G$ to be grade trade robust.

Let $\left\langle A_{1}, A_{2}, \ldots, A_{m}\right\rangle=\left\langle X_{1}, X_{2}, \ldots, X_{m}\right\rangle$, and $\left\langle B_{1}, B_{2}, \ldots, B_{m}\right\rangle=\left\langle X_{1}^{\prime}, X_{2}^{\prime}, \ldots, X_{m}^{\prime}\right\rangle$, so that we have

$$
A_{i}>_{V} B_{i}
$$

for each $i=1,2, \ldots, m$.

Let $\left\langle A_{1}^{\prime}, A_{2}^{\prime}, \ldots, A_{m}^{\prime}\right\rangle=\left\langle X_{1}^{\prime}, X_{2}^{\prime}, \ldots, X_{m}^{\prime}\right\rangle$, and $\left\langle B_{1}^{\prime}, B_{2}^{\prime}, \ldots, B_{m}^{\prime}\right\rangle=\left\langle X_{1}, X_{2}, \ldots, X_{m}\right\rangle$.

It follows that

$$
A_{i}^{\prime}<_{V} B_{i}^{\prime}
$$

for each $i=1,2, \ldots, m$, so that $G$ is not grade ${ }^{+}$trade robust.

$(\Rightarrow)$ Now, assume that $G$ is not grade ${ }^{+}$trade robust, and let

$$
A_{i}>_{V} B_{i}
$$

(for each $i=1,2, \ldots, m$ ), together with

$$
A_{i}^{\prime}<_{V} B_{i}^{\prime}
$$

(for each $i=1,2, \ldots, m$ ) witness a failure of $G$ to be grade ${ }^{+}$trade robust. Next, let

$$
\begin{aligned}
\left\langle X_{1}, X_{2}, \ldots, X_{2 m}\right\rangle & =\left\langle A_{1}, A_{2}, \ldots, A_{m}, B_{1}^{\prime}, B_{2}^{\prime}, \ldots, B_{m}^{\prime}\right\rangle \quad \text { and } \\
\left\langle X_{1}^{\prime}, X_{2}^{\prime}, \ldots, X_{2 m}^{\prime}\right\rangle & =\left\langle B_{1}, B_{2}, \ldots, B_{m}, A_{1}^{\prime}, A_{2}^{\prime}, \ldots, A_{m}^{\prime}\right\rangle .
\end{aligned}
$$

Then $\left\langle X_{1}^{\prime}, X_{2}^{\prime}, \ldots, X_{2 m}^{\prime}\right\rangle$ is obtained via trades from $\left\langle X_{1}, X_{2}, \ldots, X_{2 m}\right\rangle$, and clearly $X_{i}>_{V}$ $X_{i}^{\prime}$ for each $i=1,2, \ldots, 2 m$, so $G$ is not grade trade robust. 
Theorem 4.7 Let $G$ be any $(j, k)$ prehypergraph (simple game). Then $G$ is a weighted $(j, 2)$ prehypergraph (simple game) if and only if $G$ is trade robust.

Proof: $(\Rightarrow)$ Let $G=(N, V)$ be weighted and assume, by way of contradiction, that $G$ is not grade trade robust. Let $\left\langle X_{1}, X_{2}, \ldots, X_{m}\right\rangle$ be a sequence of $j$-partitions and $\left\langle X_{1}^{\prime}, X_{2}^{\prime}, \ldots, X_{m}^{\prime}\right\rangle$ be a second sequence obtained from the first via trades in such a way that

$$
X_{i}>_{V} X_{i}^{\prime} \text { for each } i=1,2, \ldots, m .
$$

Just as in the proof of Theorem 4.2, the condition

$$
\left|\left\{i \mid p \in X_{i}\right\}\right|=\left|\left\{i \mid p \in X_{i}^{\prime}\right\}\right|
$$

guarantees that

$$
w\left(X_{1}\right)+w\left(X_{2}\right)+\cdots+w\left(X_{m}\right)=w\left(X_{1}^{\prime}\right)+w\left(X_{2}^{\prime}\right)+\cdots+w\left(X_{m}^{\prime}\right) .
$$

Applying (11) we obtain

$$
w\left(X_{1}\right)+w\left(X_{2}\right)+\cdots+w\left(X_{m}\right)>w\left(X_{1}^{\prime}\right)+w\left(X_{2}^{\prime}\right)+\cdots+w\left(X_{m}^{\prime}\right),
$$

which contradicts (12).

$(\Leftarrow)$ Here we consider the special case $j=2$. The proof for $j>2$ appears in the appendix. As any 2-partition $A=\left({ }_{1} A,{ }_{2} A\right)$ can be identified with the coalition ${ }_{1} A$ (because the membership of ${ }_{2} A$ is completely determined by that of ${ }_{1} A$ ), this section is phrased in terms of coalitions rather than 2-partitions.

Assume $G=(N, V)$ is grade trade robust. We will define an auxiliary prehypergraph $G^{*}$ and prove that $G^{*}$ is trade robust as a (standard) prehypergraph. It then follows (by Theorem 2.4.2 of [TZ2]), that $G^{*}$ is weighted as a prehypergraph. Finally, we show that the weights for $G^{*}$ may be combined to yield weights for our original $G$.

For the grand coalition of our $G^{*}$ we will need two copies of the original grand coalition $N=\{1,2, \ldots, n\}$ of $G$. Let $N^{L}$ denote $\left\{1_{L}, 2_{L}, \ldots, n_{L}\right\}$ (our "left" copy of $N$ ), let $N^{R}$ denote $\left\{1_{R}, 2_{R}, \ldots, n_{R}\right\}$ (our "right" copy of $N$ ), and let $N^{*}=N^{L} \cup N^{R}$ be the grand coalition for $G^{*}$. For any $X \subseteq N^{*}$, let $X_{L}$ denote $\left\{i \in N \mid i_{L} \in X\right\}$ and $X_{R}$ denote $\left\{i \in N \mid i_{R} \in X\right\}$. For any $X \subseteq N$, let $X^{\wedge L}$ denote $\left\{i_{L} \mid i \in X\right\}$ and $X^{\wedge R}$ denote $\left\{i_{R} \mid i \in X\right\}$. The pregame $G^{*}$ is defined on $N^{*}$ as follows: a coalition $X$ is winning if $X_{L}>_{V} X_{R}$, losing if $X_{L}<_{V} X_{R}$, and unclassified otherwise.

We claim that $G^{*}$ is trade robust as a pregame. Suppose that $\left\langle X_{1}, X_{2}, \ldots, X_{m}\right\rangle$ is any finite sequence of $G^{*}$-winning coalitions of $N^{*}$, and a second sequence $\left\langle X_{1}^{\prime}, X_{2}^{\prime}, \ldots, X_{m}^{\prime}\right\rangle$ is obtained from the first via trades. Let $A_{i}=\left(X_{i}\right)_{L}, B_{i}=\left(X_{i}\right)_{R}, A_{i}^{\prime}=\left(X_{i}^{\prime}\right)_{L}$, and $B_{i}^{\prime}=\left(X_{i}^{\prime}\right)_{R}$. Then it is clear that if the original $X$ and $X^{\prime}$ sequences witnessed a failure of $G^{*}$ to be trade robust as a pregame (because each $X_{i}^{\prime}$ is losing in $G^{*}$ ), then 
the $A, B, A^{\prime}$, and $B^{\prime}$ sequences would witness a failure of $G$ to be grade ${ }^{+}$trade robust, contradicting our assumption.

Now suppose that $w^{*}: N^{*} \rightarrow \mathbb{R}$ and $q^{*} \in \mathbb{R}$ represent $G^{*}$ as a weighted pregame, so that $w^{*}(X) \geq q^{*}$ whenever $X$ is winning in $G^{*}$ and $w^{*}(X)<q^{*}$ whenever $X$ is losing in $G^{*}$. Define $w: N \rightarrow \mathbb{R}$ by $w(i)=w^{*}\left(i_{L}\right)-w^{*}\left(i_{R}\right)$. As pointed out immediately after the definition of weighted $(j, k)$ prehypergraphs, it suffices to show that for any two classified coalitions $X$ and $Y$ of $N$, if $X>_{V} Y$ then $w(X)>w(Y)$. Note that $X>_{V} Y$ implies $X^{\wedge L} \cup Y^{\wedge R}$ is a winning coalition of $G^{*}$, thus

$$
w^{*}\left(X^{\wedge L}\right)+w^{*}\left(Y^{\wedge R}\right) \geq q^{*} .
$$

Also, $X>_{V} Y$ implies $X^{\wedge R} \cup Y^{\wedge L}$ is a losing coalition of $G^{*}$, so that $w^{*}\left(X^{\wedge R}\right)+$ $w^{*}\left(Y^{\wedge L}\right)<q^{*}$, whence

$$
-w^{*}\left(X^{\wedge R}\right)-w^{*}\left(Y^{\wedge L}\right)>-q^{*}
$$

Adding the inequalities (13) and (14) yields $w^{*}\left(X^{\wedge L}\right)+w^{*}\left(Y^{\wedge R}\right)-w^{*}\left(X^{\wedge R}\right)-w^{*}\left(Y^{\wedge L}\right)>$ 0 , from which $w^{*}\left(X^{\wedge L}\right)-w^{*}\left(X^{\wedge R}\right)>w^{*}\left(Y^{\wedge L}\right)-w^{*}\left(Y^{\wedge R}\right)$, which is the same as $w(X)>w(Y)$, as desired.

\section{Properties for the given examples and concluding remarks}

\subsection{A representation of the UNSC as a weighted $(3,2)$ simple game}

We show that the UNSC is weighted as a $(3,2)$ simple game, and provide a simple weighted representation.

In this attempt, we will assign a common weight to all permanent members in $P$ and another common weight to all nonpermanent members in $R$. Concretely,

$$
\begin{aligned}
\left(w_{1}(a), w_{2}(a), w_{3}(a)\right) & =\left(A_{1}, A_{2}, A_{3}\right) \quad \text { if } a \in P \\
\left(w_{1}(b), w_{2}(b), w_{3}(b)\right) & =\left(B_{1}, B_{2}, B_{3}\right) \quad \text { if } b \in R
\end{aligned}
$$

where $A_{1}\left(B_{1}\right)$ denotes the weight cast by the player $a(b)$ if that player votes "yes," $A_{2}\left(B_{2}\right)$ denotes the weight cast by the player $a(b)$ if that player abstains, and $A_{3}\left(B_{3}\right)$ denotes the weight cast by the player $a(b)$ if that player votes "no."

We will also normalize these weights by setting $w_{\text {abstain }}(p)=0$ for each of the two types of players. Consequently $A_{2}=B_{2}=0 ; A_{1}, B_{1} \geq 0$; and $A_{3}, B_{3} \leq 0$. Because for nonpermanent members, voting "no" is tantamount to abstaining, we will also assume that $B_{3}=0$.

By considering types of minimal winning 3-partitions and maximal losing 3-partitions we reduce the question of weightedness to that of the consistency of the following system of inequalities: 


$$
\begin{aligned}
5 A_{1}+4 B_{1} & \geq q \\
4 A_{1}+5 B_{1} & \geq q \\
3 A_{1}+6 B_{1} & \geq q \\
2 A_{1}+7 B_{1} & \geq q \\
A_{1}+8 B_{1} & \geq q \\
9 B_{1} & \geq q \\
4 A_{1}+10 B_{1}+A_{3} & <q \\
5 A_{1}+3 B_{1} & <q \\
4 A_{1}+4 B_{1} & <q \\
3 A_{1}+5 B_{1} & <q \\
2 A_{1}+6 B_{1} & <q \\
A_{1}+7 B_{1} & <q \\
8 B_{1} & <q \\
A_{1} \geq 0, A_{3} & \leq 0 \\
B_{1} & \geq 0 .
\end{aligned}
$$

A solution of this system is: $q=9$, and

$$
\begin{aligned}
& \left(A_{1}, A_{2}, A_{3}\right)=(1,0,-6) \\
& \left(B_{1}, B_{2}, B_{3}\right)=(1,0,0) .
\end{aligned}
$$

In this system of weights, there is no difference between the "yes" weights of permanent and nonpermanent members, but the relative sizes of their "no" weights strongly reflect the decisiveness of permanent members in rendering a negative decision in the UNSC. Finally, let us note that the system presented here is strongly weighted.

The corresponding quota and "step" weights for the second weightedness notion we introduced are

$$
q^{\prime}=38, \quad\left(d_{1}(a), d_{2}(a)\right)=(1,6) \text { and }\left(d_{1}(b), d_{2}(b)\right)=(1,0)
$$

and this representation is the unique integer minimal (component by component) step weight representation. Notice this latter representation corresponds to infinitely many "level" weight representations; (15) is one of these.

\subsection{Two of the Senate games are weighted, and two are not}

First we take up the three games that take no account of quorums. It is straightforward to see that the game $V_{B}$ for passing bills in the Senate is weighted; each senator $s$ is assigned weights of

$$
\left(w_{1}(s), w_{2}(s), w_{3}(s)\right)=(1,0,-1)
$$

and the vice president is assigned weights of

$$
\left(w_{1}(v p), w_{2}(v p), w_{3}(v p)\right)=(0.5,0,0)
$$


with the quota set at 0.1 , for example.

The game $V_{T}$ considered in (9) for treaty ratification is also weighted, with the weight of each senator given by

$$
\left(w_{1}(s), w_{2}(s), w_{3}(s), w_{4}(s)\right)=(1,0,-2,-2)
$$

and the quota set at 0 . With the alternate version $V_{T^{\prime}}$ (in which the treaty goes unratified when all senators are absent) defined as in (10), the senator's weights and the quota are slightly different:

$$
\left(w_{1}(s), w_{2}(s), w_{3}(s), w_{4}(s)\right)=(1+\epsilon, 0,-2,-2)
$$

and the quota set at $2 \epsilon$, with $\epsilon$ being any number in the interval $(0,1 / 63)$. In both cases the vice-president's weights are all zeros.

The $(3,3)$ simple game $V_{v p}$ for electing a vice-president is not weighted, as we show by a failure of grade trade robustness. Suppose we begin with ordered partitions

$$
\begin{gathered}
\left({ }_{1} A,{ }_{2} A,{ }_{3} A\right) \text { with }{ }_{1} A=\{1,2, \ldots, 51\},{ }_{2} A=\emptyset, \text { and }{ }_{3} A=\{v p, 52,53, \ldots, 100\} ; \text { and } \\
\left({ }_{1} B,{ }_{2} B,{ }_{3} B\right) \text { with }{ }_{1} B=\emptyset,{ }_{2} B=\{50,51, \ldots, 100\} \text { and }{ }_{3} B=\{v p, 1,2, \ldots, 49\} .
\end{gathered}
$$

(Here the senators are numbered from 1 to 100.) Then $V_{v p}(A)=a$ wins, while $V_{v p}(B)=$ inconclusive. Now imagine that senators number 50 and 51 in ${ }_{1} A$ switch their membership to ${ }_{2} A$; these same two senators belonged to ${ }_{2} B$ and make the corresponding switch to ${ }_{1} B$. At the same time all 49 senators numbered 52 through 100 belonging to ${ }_{3} A$ switch their membership to ${ }_{2} A$; these same 49 senators all belonged to ${ }_{2} B$ and make the corresponding switch to ${ }_{3} B$. After these switches the ordered partitions have been transformed as follows:

$$
\begin{aligned}
& \left({ }_{1} A^{\prime},{ }_{2} A^{\prime},{ }_{3} A^{\prime}\right) \text { with }{ }_{1} A^{\prime}=\{1,2, \ldots, 49\},{ }_{2} A^{\prime}=\{50,51, \ldots, 100\},{ }_{3} A^{\prime}=\{v p\} ; \text { and } \\
& \left({ }_{1} B^{\prime},{ }_{2} B^{\prime},{ }_{3} B^{\prime}\right) \text { with }{ }_{1} B^{\prime}=\{50,51\},{ }_{2} B^{\prime}=\emptyset \text { and }{ }_{3} B^{\prime}=\{v p, 1,2, \ldots, 49,52, \ldots, 100\}
\end{aligned}
$$

Then $V_{v p}\left(A^{\prime}\right)=$ inconclusive, while $V_{v p}\left(B^{\prime}\right)=b$ wins, so that

$$
V_{v p}(A)>V_{v p}\left(A^{\prime}\right) \text { and } V_{v p}(B)>V_{v p}\left(B^{\prime}\right)
$$

a violation of grade trade robustness.

As for our hypergraph $V_{B Q}$ for passing bills with quorum incorporated, the inconsistent effect (discussed in Section 3.2.4) of a single voter switching from "no" to "abstain" immediately guarantees that the game is not weighted. This can easily be shown by exhibiting a failure of grade trade robustness, but it should also be clear that any weighted game has some particular step weight corresponding to a switch from "no" to "abstain" for the particular voter who switches, and this step weight is either nonnegative (in which case a switch from "no" to "abstain" can never decrease the output level of approval) or nonpositive (in which case this switch can never strictly increase the output level); that is, a weighted game can never exhibit the inconsistency observed in $V_{B Q}$. 


\subsection{The non-weightedness of Hsiao and Raghavan's example}

Consider the following four 4-partitions:

$$
\begin{gathered}
(\emptyset, N-D,\{1,2\},\{3, \ldots, 10\}), \\
(\{1,27, \ldots, 50\}, \emptyset, \emptyset,\{2, \ldots, 26\}), \\
(\{1\},\{13, \ldots, 50\},\{2\},\{3, \ldots, 12\}), \\
(\{27, \ldots, 50\},\{11,12\},\{1\},\{2, \ldots, 10,13, \ldots, 26\}) .
\end{gathered}
$$

These partitions are respectively winning, winning, losing and losing. After a trade the first and the second 4-partitions are converted into the third and the fourth 4-partitions, witnessing a failure of $G$ to be grade trade robust.

\subsection{Grading Systems}

The American grading system is clearly presented as a weighted $(10,10)$-game, and it is easy to see that it is strongly weighted.

For the Catalan grading system, from the system of linear inequalities formed by $i$-minimal and $(i+1)$-maximal 3 -partitions, $(i=1,2,3)$ we get the following weighted representation (renormalizing at the middle level):

$$
\begin{array}{cc}
\left(w_{1}(\mathrm{th}), w_{2}(\mathrm{th}), w_{3}(\mathrm{th})\right) & =(1,0,-3) \\
\left(w_{1} \text { (pro) }, w_{2} \text { (pro) }, w_{3}(\text { pro })\right) & =(1,0,-2) \\
\left.\left(w_{1} \text { (pra) }\right), w_{2}(\text { pra }), w_{3}(\text { pra })\right) & =(2,0,-1)
\end{array}
$$

and quotas:

$$
q_{0}=3, \quad q_{1}=2, \quad q_{2}=1 \text {. }
$$

\subsection{Concluding remarks}

Our approach has been to take some selected topics in the study of simple games, and extend them to the broader context of $(j, k)$ simple games, which are games with several linearly ordered input states and several linearly ordered output states. We were initially motivated by recent interest in modifying ordinary simple games to take account of abstention - that is, by $(3,2)$ simple games - and by the question of what "weighted voting" should mean when abstentions are allowed. But the existence of real examples with larger $j$ and $k$ values, and the ease with which our proposed definition of weighted voting with abstention extends to these higher values, suggests to us the naturalness of the broader context.

Certainly, further generalization would be possible. For example, one could remove the linear ordering on the input or on the output set, moving the model in the direction of Social Choice theory. As an hypothetical example, if the Senate were allowed 
to choose from among the 3 highest electoral college candidates, it would be more clear why there is no natural order to the inputs, or to the outputs "elect $a$," "elect $b$," "elect $c$," and "inconclusive." While this certainly suggests the importance of a different model without ordering of levels, it seems unlikely that coincidence alone can explain the number of examples for which orderings on the sets of inputs and outputs are clearly implicit in the situation being modeled.

Within $(j, k)$ games, several directions for further research suggest themselves. One broad area is that of strategic notions. Do point-valued solutions for simple games (such as Shapley-Shubik Index, Banzhaf index, nucleolus, etc.) or set-valued solutions (such as core, bargaining set, kernel, etc.) have useful extensions to $(j, k)$ games?

A second direction worth considering is that of structural notions for $(j, k)$ games. Is there an analogue of linear games (simple games with an individual desirability relation that is total) in the context of $(j, k)$ games, and, if so, do there exist classification theorems for linear $(j, k)$ games that are in the same spirit as those of $\mathrm{Hu}[\mathrm{Hu}]$ and of Carreras and Freixas [CF] for ordinary linear simple games? Is there an analogue of dimension theory (see [TZ2]) for $(j, k)$ simple games, in which every game can be viewed as an intersection of weighted games?

\section{Appendix}

Theorem 6.1 If $G$ is a grade trade robust $(j, k)$ prehypergraph (simple game) with $j>2$, then $G$ is a weighted prehypergraph (simple game).

Proof: Assume $G=(N, V)$ is grade trade robust. We will define an auxiliary $(j, 2)$ prehypergraph $G^{*}$, and prove that $G^{*}$ is trade robust as a $(j, 2)$ prehypergraph. It then follows, by Theorem 4.4 applied to $(j, 2)$ prehypergraphs, that $G^{*}$ is weighted as a $(j, 2)$ prehypergraph. Finally, we show that the weights for $G^{*}$ may be combined to yield weights for our original $G$.

For $G^{*}$, we will need two disjoint copies of the original grand coalition $N=\{1,2, \ldots, n\}$ of $G$. Let $N^{L}$ denote $\left\{1_{L}, \ldots, n_{L}\right\}$ (our "left" copy of $N$ ), let $N^{R}$ denote $\left\{1_{R}, \ldots, n_{R}\right\}$ (our "right" copy), and let $N^{*}=N^{L} \cup N^{R}$ be the grand coalition for $G^{*}$. For any $X \subseteq N^{*}$ let $X_{L}$ denote $\left\{i \in N \mid i_{L} \in X\right\}$ and $X_{R}$ denote $\left\{i \in N \mid i_{R} \in X\right\}$. For any $X \in{ }^{j}\left(N^{*}\right)$ let $X_{L}$ denote $\left(\left({ }_{1} X\right)_{L},\left({ }_{2} X\right)_{L}, \ldots,\left({ }_{j} X\right)_{L}\right) \in{ }^{j} N$ and let $X_{R}$ denote $\left(\left({ }_{1} X\right)_{R},\left({ }_{2} X\right)_{R}, \ldots,\left({ }_{j} X\right)_{R}\right) \in{ }^{j} N$. For any $X \subseteq N$, let $X^{\wedge L}$ denote $\left\{i_{L} \mid i \in X\right\}$ and $X^{\wedge R}$ denote $\left\{i_{R} \mid i \in X\right\}$. For any $X, Y \in{ }^{j} N$, let $X^{\wedge L}$ denote $\left(\left({ }_{1} X\right)^{\wedge L},\left({ }_{2} X\right)^{\wedge L}, \ldots,\left({ }_{j} X\right)^{\wedge L}\right)$, let $X^{\wedge R}$ denote $\left(\left({ }_{1} X\right)^{\wedge R},\left({ }_{2} X\right)^{\wedge R}, \ldots,\left({ }_{j} X\right)^{\wedge R}\right)$ and let $X^{\wedge L}{ }^{j} \cup Y^{\wedge R}$ denote $\left(\left({ }_{1} X\right)^{\wedge L} \cup\right.$ $\left.\left({ }_{1} Y\right)^{\wedge R},\left({ }_{2} X\right)^{\wedge L} \cup\left({ }_{2} Y\right)^{\wedge R}, \ldots,\left({ }_{j} X\right)^{\wedge L} \cup\left({ }_{j} Y\right)^{\wedge R}\right)$. The $(j, 2)$ prehypergraph $G^{*}$ is defined on $N^{*}$ as follows: a $j$-partition $X$ is winning if $X_{L}>_{V} X_{R}$, losing if $X_{L}<_{V} X_{R}$, and unclassified otherwise.

We claim that $G^{*}$ is trade robust as a $(j, 2)$ prehypergraph. Suppose that $\left\langle X_{1}, X_{2}, \ldots, X_{l}\right\rangle$ is any finite sequence of $G^{*}$-winning $j$-partitions of $N^{*}$, and a sec- 
ond sequence $\left\langle X_{1}^{\prime}, X_{2}^{\prime}, \ldots, X_{l}^{\prime}\right\rangle$ is obtained from the first via trades. Let $A_{i}=\left(X_{i}\right)_{L}$, $B_{i}=\left(X_{i}\right)_{R}, A_{i}^{\prime}=\left(X_{i}^{\prime}\right)_{L}$, and $B_{i}^{\prime}=\left(X_{i}^{\prime}\right)_{R}$. Then it is clear that if the original $X$ and $X^{\prime}$ sequences witnessed a failure of $G^{*}$ to be trade robust as a $(j, 2)$ prehypergraph (because each $X_{i}^{\prime}$ is losing in $G^{*}$ ), then the $A, B, A^{\prime}$, and $B^{\prime}$ sequences would witness a failure of $G$ to be grade ${ }^{+}$trade robust, contradicting our assumption.

Now suppose that $w^{*}=\left(w_{1}^{*}, \ldots, w_{j}^{*}\right)$ where $w_{s}^{*}: N^{*} \rightarrow \mathbb{R}$ for each $s$ with $1 \leq s \leq j$ and $q^{*} \in \mathbb{R}$ realize $G^{*}$ as a weighted $(j, 2)$ prehypergraph, so that $w^{*}(X) \geq q^{*}$ whenever $X$ is winning in $G^{*}$ and $w^{*}(X)<q^{*}$ whenever $X$ is losing in $G^{*}$. Define $w_{s}: N \rightarrow \mathbb{R}$ by $w_{s}(i)=w_{s}^{*}\left(i_{L}\right)-w_{s}^{*}\left(i_{R}\right)$ for each $s$ with $1 \leq s \leq j$ and let $w=\left(w_{1}, \ldots, w_{j}\right)$. As pointed out immediately after Definition 2.5, it suffices to show that for any two classified $j$-partitions $X$ and $Y$ of $N$, if $X>_{V} Y$ then $w(X)>w(Y)$.

Note that $X>_{V} Y \Rightarrow X^{\wedge L} j \cup Y^{\wedge R}$ is a winning $j$-partition of $G^{*}$, so that

$$
w^{*}\left(X^{\wedge L}\right)+w^{*}\left(Y^{\wedge R}\right) \geq q^{*}
$$

Also, $X>_{V} Y \Rightarrow X^{\wedge R} j \cup Y^{\wedge L}$ is a losing $j$-partition of $G^{*}$, so that

$$
w^{*}\left(X^{\wedge R}\right)+w^{*}\left(Y^{\wedge L}\right)<q^{*}
$$

whence

$$
-w^{*}\left(X^{\wedge R}\right)-w^{*}\left(Y^{\wedge L}\right)>-q^{*}
$$

Adding the inequalities (16) and (17) yields

$w^{*}\left(X^{\wedge L}\right)+w^{*}\left(Y^{\wedge R}\right)-w^{*}\left(X^{\wedge R}\right)-w^{*}\left(Y^{\wedge L}\right)>0$, from which

$w^{*}\left(X^{\wedge L}\right)-w^{*}\left(X^{\wedge R}\right)>w^{*}\left(Y^{\wedge L}\right)-w^{*}\left(Y^{\wedge R}\right)$, which is the same as $w(X)>w(Y)$, as desired.

Finally, note that if $G$ is a $(j, k)$ simple game, then as $G$ is monotonic we can, by Theorem 2.7, adjust the weight functions to satisfy that for each $x \in N, w_{1}(x) \geq w_{2}(x) \geq$ $\ldots \geq w_{j}(x)$.

\section{References}

[ACM] Amer, R., Carreras, F. and Magaña, A. (1998), The Banzhaf-Coleman index for games with $r$ alternatives, Optimization 44, 175-198.

[Bo1] Bolger, E. M. (1986), Power indices for multicandidate voting games, Int. Journal of Game Theory 14, 175-186.

[Bo2] Bolger, E. M. (1993), A value for games with $n$ players and $r$ alternatives, Int. Journal of Game Theory 22, 319-334. 
[Bo3] Bolger, E. M. (1993), A consistent value for games with $n$ players and $\boldsymbol{r}$ alternatives, Int. Journal of Game Theory 29, 93-99.

[BS] Braham, M. and Steffen, F. (2001), Voting power in games with abstentions, Forthcoming in Jahrbuch für Neue Politische Ekonomie 20.

[CF] Carreras, F. and Freixas, J. (1996), Complete simple games, Mathematical Social Science 32, 139-155.

[deF] de Finetti, B. (1937), La prévision, ses lois logiques, ses sources subjectives, Annales de l'Institut. H. Poincaré, 7, 1-68 (Translated in: Kyburg, H. E. Jr., and Smokler H. E., eds., (1964): Studies in Subjective Probability, Wyley, New York).

[EL] Einy, E. and Lehrer, E. (1989), Regular simple games, Int. Journal of Game Theory $18,195-207$.

[FM1] Felsenthal, D. S. and Machover, M. (1997), Ternary voting games, Int. Journal of Game Theory 26, 335-351.

[FM2] Felsenthal, D. S. and Machover, M. (2001), Models and reality: the curious case of the absent abstention, in Holler M. J. and Owen G. (eds.) Power Indices and Coalition Formation, Dordrecht.

[FM3] Felsenthal, D. S. and Machover M. (1998), The Measurement of Voting Power: Theory and Practice, Problems and Paradoxes. Edward Elgar Publishing Limited.

[Fas] Fassbender, B. (1998), UN Security Council Reform and the Right of Veto; The Hague: Kluwer Law International.

[Fis1] Fishburn, P. C. (1973), The Theory of Social Choice; Princeton: Princeton University Press.

[Fis2] Fishburn, P. C. (1986), The axioms of subjective probability, Statistical Science 1, 335-345.

[HR] Hsiao C. R. and Raghavan, T. E. S. (1993), Shapley value for multichoice cooperative games I, Games and Economic Behavior 5, 240-256.

[Hu] Hu, S. T. (1961), Threshold Logic. Berkeley: University of California Press.

[Is1] Isbell, J. R. (1956), A class of majority games. Quarterly Journal of Math, 2d ser., 7, 183-187.

[Is2] Isbell, J. R. (1958), A class of simple games, Duke Math. Journal 25, 423-439.

[KPS] Kraft, C. H., Pratt J. W., and Seidenberg A. (1959), Intuitive probability on finite sets, Annals of Mathematical Statistics 30, 408-419.

[Lin1] Lindner, I. (2001), Probabilistic characterizations of power indices with abstentions, Mimeo. 
[Lin2] Lindner, I. (2001), Multi-partition games, Mimeo.

[List] List, C. (2001), A note on special majority voting from an epistemic perspective, Preprint.

[Mad] Madison, J., et al. The Constitution of the United States of America; at http://www.nara.gov/exhall/charters/constitution/constitution.html

[Mag] Magaña, A. (1996), Formación de coaliciones en los juegos cooperativos y juegos con múltiples alternativas, Ph.D. Thesis, Polytechnic University of Catalonia.

[MP] Maschler, M. and Peleg, B. (1966), A characterization, existence proof, and dimension bounds for the kernel of a game, Pacific Journal of Math. 18, 289-328.

[SRS] The Standing Rules of the Senate; http://rules.senate.gov/senaterules/menu.htm

[SS] Shapley, L. S. and Shubik, M. (1954), A method for evaluating the distribution of power in a committee system, American Political Science Review 48, 787-792.

[Sim] Simma, B. (1994), (in collaboration with Hermann Mosler, Albrecht Randelzhofer, Christian Tomuschat, and Rädiger Wolfrum). The charter of the United Nations: A Commentary. Oxford: Oxford University Press, 1994.

[Str] Straffin, P. D. (1983), Power indices in politics, in Brams S. J., Lucas W. F. and Straffin P. D. (eds.) Political and Related Models, Berlin, 256-321.

[TZ1] Taylor, A. D. and Zwicker, W. S. (1992), A characterization of weighted voting, Proceedings of the American Mathematical Society 115, 1089-1094.

[TZ2] Taylor, A. D. and Zwicker, W. S. (1999), Simple games: desirability relations, trading, and pseudoweightings, Princeton University Press, New Jersey. 